\title{
Elaiophylin Targets EIF4B to Regulate the \\ Proliferation, Invasion, and Apoptosis of Esophageal Squamous Cell Carcinoma via the PI3K/AKT Signaling Pathway
}

\section{Gao Lijuan}

Wuhan University First Clinical College: Wuhan University Renmin Hospital https://orcid.org/00000002-5343-5396

\section{Yongshun Chen ( $\sim 2018203020082 @ w h u . e d u . c n)$}

Wuhan University Affiliated First Clinical Hospital: Wuhan University Renmin Hospital https://orcid.org/0000-0001-5299-8544

\section{Bin Li}

Wuhan University Affiliated First Clinical Hospital: Wuhan University Renmin Hospital

\section{Research Article}

Keywords: Esophageal squamous cell carcinoma, PI3K/AKT, EIF4B, Migration, Proliferation, Apoptosis

Posted Date: October 27th, 2021

DOI: https://doi.org/10.21203/rs.3.rs-604592/v2

License: () (i) This work is licensed under a Creative Commons Attribution 4.0 International License. Read Full License 


\section{Abstract \\ Background}

Esophageal cancer remains a dominating cause of cancer-associated death and has shown a sharp increase of more than 6 -fold increase rates all over the world. It is divided into two main pathological types: esophageal squamous cell carcinoma (ESCC) and esophageal adenocarcinoma (EAC). Most of the patients are diagnosed at the advanced stage with distant metastasis and poor prognosis. Thus, it is urgent for us to identify new more effective drugs to improve the prognosis of patients with esophageal cancer. Elaiophylin is a novel autophagy inhibitor at the late stage, which is a C2 symmetric macrolides separated from Streptomyces Niger. It was proved to have anti-tumor ability of various of cancers, yet little is known whether it could inhibit the progression of esophageal squamous cancer. The study is aimed to explore the effect of Elaiophylin on ESCC.

\section{Methods}

The protein expression level was detected by western blot assay. The viability of ESCC cells was detected by CCK8 assay. The proliferation rate was measured by ki67 and BrdU immunofluorescent staining. The migratory ability of ESCC cells was identified by transwell assay. The apoptosis rate of ESCC cells was explored by Annexin V/PI analysis. And the genes might be regulated by Elaiophylin was analyzed by RNA-seq assay and human cancer pathway phosphorylation antibody array then verified by RT-qPCR and western blot assay. The experiments in vivo were conducted by subcutaneous into tumor in BALB/C-Nu mice.

\section{Results}

In the study, we firstly found that the Elaiophylin was an autophagy inhibitor at the late stage in ESCC cells which is consistent with the previous studies. In addition, Elaiophylin can inhibit the proliferation and migration of ESCCs, and accelerate its apoptosis. And the activity of PI3K/AKT pathway was inhibited by Elaiophylin, the mRNA and protein expression level of EIF4B was both downregulated by Elaiophylin. In addition, When PI3K/AKT and autophagy signaling pathway was inhibited, the expression of EIF4B was subsequently abrogated. Mechanistically, silencing of EIF4B could inhibit the proliferation and migration of ESCC cells and promoted apoptosis, which could be rescued by EIF4B-OE. Moreover, EIF4B overexpression suppressed the apoptosis, while increased the proliferation and migration of ESCC cells which resulted from Elaiophylin. Also, the EIF4B overexpression reversed the autophagic inhibition caused by Elaiophylin. Finally, Elaiophylin could inhibit the proliferation of esophageal cancer in vivo compared to the control group.

\section{Conclusions}


Together, our results indicated that Elaiophylin is an autophagy inhibitor governing growth and migratory ability of ESCC cells through PI3K/AKT signaling pathway and inhibit the proliferation of esophageal tumor in vivo. simultaneously, and identified EIF4B as a target of Elaiophylin, which might provide a new therapeutic strategy for esophageal squamous cell carcinoma.

\section{Introduction}

Esophageal cancer remains a dominating cause of cancer-associated death and has shown a sharp increase of more than 6 -fold increase rates all over the world. It is divided into two main pathological types: esophageal squamous cell carcinoma (ESCC) and esophageal adenocarcinoma (EAC). Among them, the incidence of ESCC accounts for about $90 \%$, which is the main position ${ }^{1}$. Moreover, ESCC has a high prevalence in East Asia, eastern and southern Africa, and southern European ${ }^{2,3}$. Because the early symptoms of esophageal cancer are hidden, most of the patients are diagnosed at the advanced stage with distant metastasis ${ }^{4}$. After surgical resection or radiotherapy and chemotherapy, due to the strong invasiveness of ESCC and the resistance of tumor tissue to drugs, the recurrence and metastasis rate is high, the prognosis is poor ${ }^{5,6}$. Though the treatment has been improved, the prognosis is still very poor with overall survival rate at 5 year was $15 \%-25 \%$. Thus, it is urgent to identify new more effective drugs to improve the prognosis of patients with esophageal cancer.

Elaiophylin is a novel autophagy inhibitor at the late stage, which is a C2 symmetric macrolides separated from Streptomyces Niger. It was firstly proved to have anti-tumor ability of ovarian cancer ${ }^{7}$ as well as multiple myeloma with mutant $\mathrm{TP}^{2} 3^{8}$, yet little is known whether it could inhibit the progression of esophageal squamous cancer. EIF4B is an important component of eukaryotic transcription initiation complex, which can be recruited to the $5^{\prime}$ untranslated region ( $5^{\prime} \mathrm{UTR}$ ) of the mRNA. It was deemed to enhance the activity of elF4A helicase to activate the function of elF4F helicase ${ }^{9}$. Moreover, it can be regulated and phosphorylated by proto-oncogenic signaling pathways such as PI3K/mTOR, AKT. Moreover, elF4B was demonstrated to regulate the translation of mRNAs which can regulate the cell proliferation and survival at the structured $5^{\prime} \mathrm{UTR}^{10}$. In recent years, elF4B was suggested to play an important role in the progression of a various cancer cells ${ }^{11,12}$.

Here, we report that Elaiophylin could inhibit the proliferation, migration of ESCC cells and provoke its apoptosis by targeting the elF4B via PI3K/AKT signaling pathway. In the study, we firstly confirmed that the Elaiophylin was an autophagy inhibitor in ESCC cells which is consistent with the previous studies. In addition, Elaiophylin can inhibit the proliferation and migration of ESCCs, and accelerate its apoptosis. Then, using human cancer pathway phosphorylation antibody array and RNA-seq analysis to identify the proteins and genes which were regulated by Elaiophylin and verify them by western blot and RT-qPCR assay. Finally, we found the activity of PI3K/AKT pathway was inhibited by Elaiophylin. And the mRNA and protein expression level of EIF4B was both downregulated by Elaiophylin. The EIF4B can be regulated by PI3K/AKT and autophagy signaling pathway. And knockdown of EIF4B could inhibit the proliferation and migration and promote apoptosis of ESCC cells, which could be rescued by EIF4B-OE. We also found 
that the elF4B overexpression could block the phenotype which was resulted from Elaiophylin. In addition, Elaiophylin could inhibit the proliferation of esophageal tumor in vivo compared to the control group. Our results suggested that the Elaiophylin might serve as a promising therapeutic medicine for esophageal squamous cell carcinoma.

\section{Materials And Methods}

\section{Cell culture, antibodies and reagents}

Eca109 and KYSE450 cell lines were obtained from ATCC (American Type Culture Collection), and cultured in RPMI-1640 (Gibco)medium supplemented with 10\% fetal bovine serum (Gibco), 100U/ml penicillin and $100 \mu \mathrm{g} / \mathrm{ml}$ streptomycin at $37^{\circ} \mathrm{C}$ in a humidified air of $5 \% \mathrm{CO}_{2}$ saturation. Both cell lines were authenticated by STR and tested for mycoplasma contamination. EIF4B Rabbit Polyclonal antibody (17917-1-AP), AKT Rabbit Polyclonal antibody (10176-2-AP), Phospho-AKT (Ser473) Mouse Monoclonal antibody (66444-1-Ig), PI3 Kinase p85 Alpha Mouse Monoclonal antibody (60225-1-lg), LC3 Rabbit Polyclonal antibody (14600-1-AP), P62/SQSTM1 Rabbit Polyclonal antibody (18420-1-AP), E-cadherin Rabbit Polyclonal antibody (20874-1-AP), BAX Rabbit Polyclonal antibody (50599-2-lg), BCL2 Rabbit Polyclonal antibody (12789-1-AP) were purchased from Proteintech. The Phospho-PI3KP85a/ $/ / \beta-$ Y467/Y199/Y464 Rabbit Phosphorylated Antibodies (AP0854), ACTB Rabbit mAb (AC026), GAPDH Mouse mAb (AC002) were purchased from ABclonal. The Cell Counting Kit-8 and Crystal Violet Staining Solution were purchased from Beyotime. The FITC Annexin V Apoptosis Detection Kit I (556547) was purchased from BD Pharmingen. The Corning Costar Transwell, pore size $8.0 \mu \mathrm{m}$ was purchased from Corning.

\section{Protein extraction and Western blot assay}

The cells which pretreatment with Elaiophylin or transfection with vectors were collected by $200 \mu \mathrm{L}$ loading buffer followed by warming at $100^{\circ} \mathrm{C}$ for $10 \mathrm{~min}$. The protein concentration in the supernatants was detected by standard bicinchoninic acid (BCA) method. The proteins were loaded onto a $10 \%$ sodium dodecyl sulfate polyacrylamide gel, and proteins were separated by electrophoresis. Then, the proteins were transferred onto a polyvinylidene fluoride (PVDF) membrane with a constant current of $200 \mathrm{~mA}$ at $2 \mathrm{~h}$ and blocked with $10 \%$ non-fat milk at room temperature for $1 \mathrm{~h}$. Afterwards, recycling the milk and washing the membrane with Tris-buffered saline with $0.1 \% \mathrm{v}: \mathrm{v}$ Tween 20 , the membranes were incubated with the anti-PI3K, anti-p-PI3K, anti-AKT, anti-p-AKT, anti-EIF4B, anti-E-cadherin, anti-Bax, anti-Bcl-2 antibodies overnight at $4^{\circ} \mathrm{C}$. After washing the membranes with TBST $10 \mathrm{~min}$ three times, the membranes were subsequently incubated with the second antibody horseradish peroxidase-conjugated immunoglobin $\mathrm{G}$ for $1 \mathrm{~h}$ at room temperature. The results of the expression level of proteins were detected by enhanced chemiluminescence (ECL) kit and developed by $x$-ray film in the darkroom. And the semi-quantitative analysis of relative expression of proteins normalized to GAPDH or ACTIN was conducted by Image J Fiji software system. 


\section{Immunofluorescence cell staining of Ki67 and BrdU}

After cell counting, we added 50,000 cells to each climbing piece. After $24 \mathrm{~h}$, wash the cells with PBS three times and fixed them with $4 \%$ paraformaldehyde and permeabilized them with $0.5 \%$ Triton X-100 for $20 \mathrm{~min}$ at the room temperature. Then blocked the cells with $5 \%$ goat serum for $30 \mathrm{~min}$, and followed by incubating with primary antibodies overnight at $4{ }^{\circ} \mathrm{C}$. Next day, recycled the primary antibodies and washed the cells with PBS three times. Then incubated the cells with fluorescein isothiocyanate-labeled secondary antibodies for $1 \mathrm{~h}$ at the room temperature. Whereafter, washed the cells with PBS three times once again and incubated with DAPI for $2 \mathrm{~min}$. Following by washing with PBS one time, used absorbent paper to absorb the liquid on the slide and mounted the slide with a mounting solution containing antifluorescence quencher and then observed and collected the image under a laser scanning confocal microscope.

\section{Cell transfection}

Short hairpin RNA (shRNA) for EIF4B, ATG5, ATG7 and EIF4B expressing plasmids were transiently transfected into Eca109 and KYSE450 cell lines using Lipofectamine ${ }^{\mathrm{TM}} 3000$ (Invitrogen, Carlsbad, CA, USA) according to the manufacturer's instructions. The sequences of EIF4B shRNA were shEIF4B-1 forwards,5'-CCGGTGTTACAGAAGAGTCAATTAACTCGAGTTAATTGACTCTTCTGTAACATTTTTG-3' and reverse, 5'- AATTCAAAAATGTTACAGAAGAGTCAATTAACTCGAGTTAATTGACTCTTCTGTAACA-3'. ShEIF4B-2 forwards, 5'-

CCGGGACAAGTATCGAGATCGTTATCTCGAGATAACGATCTCGATACTTGTCTTTTTG-3' and reverse 5'AATTCAAAAAGACAAGTATCGAGATCGTTATCTCGAGATAACGATCTCGATACTTGTC-3'. The sequences of shATG5-1 were forwards 5'-

CCGGCCTGAACAGAATCATCCTTAACTCGAGTTAAGGATGATTCTGTTCAGGTTTTTG-3' and revers 5'AATTCAAAAACCTGAACAGAATCATCCTTAACTCGAGTTAAGGATGATTCTGTTCAGG-3'. ShATG5-2 were forward 5'-CCGGCCTTTCATTCAGAAGCTGTTTCTCGAGAAACAGCTTCTGAATGAAAGGTTTTTG-3' and reverse 5'-AATTCAAAAACCTTTCATTCAGAAGCTGTTTCTCGAGAAACAGCTTCTGAATGAAAGG-3'. The sequences of shATG7-1 were forwards 5'-

CCGGGCCTGCTGAGGAGCTCTCCATCTCGAGATGGAGAGCTCCTCAGCAGGCTTTTTG-3'and reverse 5'ATGGAGAGCTCCTCAGCAGGCGAGCTCGCCTGCTGAGGAGCTCTCCATAAAAACTTAA-3'. ShATG7-2 were forward 5'- CCGGCCCAGCTATTGGAACACTGTACTCGAGTACAGTGTTCCAATAGCTGGGTTTTTG-3'and reverse 5'- TACAGTGTTCCAATAGCTGGGGAGCTCCCCAGCTATTGGAACACTGTAAAAAACTTAA-3'.

\section{Human cancer pathway phosphorylation antibody array}

Tumor signaling pathway phosphorylation broad-spectrum screening antibody array (CSP100, Full Moon) were brought from $\mathrm{H}$. Wayen and applied following the manufacturer's instructions. After extracting the protein, which quantitated by BCA assay, it was labled by Biotin/DMF. The chip was sealed with blocking 
buffer, and washed by ddH2O, following by incubating with Protein Coupling Mix. Finally, the chip was incubated with Cy3-Streptavidin Solution, and scanned by Agilent SureScan Dx Microarray Scanner at $532 \mathrm{~nm}$.

\section{Cell counting kit (CCK)-8 assay}

The Eca109 and KYSE450 cells were seeded in 96 well plates at a density of 8,000 and 10,000 cell per well respectively. All cells were divided into blank groups, untreated control groups and different concentrations of Elaiophylin for $12 \mathrm{~h}$ groups. All samples were incubated in an $5 \% \mathrm{CO}_{2}$, humidified atmosphere at $37^{\circ} \mathrm{C}$. Every group was established three holes. Subsequently, $10 \mu \mathrm{L}$ CCK-8 solution was added to each well with $100 \mu \mathrm{L}$ medium and incubated for $30 \mathrm{~min}$ in an $5 \% \mathrm{CO}_{2}$, humidified atmosphere at $37^{\circ} \mathrm{C}$. Finally, the absorbance was detected at the wavelength by using microplate reader.

\section{Transwell migration assay}

When the esophageal squamous carcinoma cells were grown to $80 \%-90 \%$ confluence, the cultured medium was removed, following washed with pre-cold PBS, we starved the cells with EBSS for $3 \mathrm{~h}$. Then, the cells were digested by $0.25 \%$ trypsin, and resuspended in starvation medium with non-FBS. After cell counting, $1 \times 105$ cells were added on the top of the chambers and filled the bottom room of the chambers with medium containing 10\% FBS. After cultivated for 24h, cells migrated to the other side of the insert. Subsequently, the $500 \mu \mathrm{L}$ PFA was added to the insert of chamber to fix the cells, followed by staining with crystal violet for $10 \mathrm{~min}$ and dried at the room temperature. Finally, counted the number of cells passing through the chamber in each experimental group under the microscope, an average of 6 fields per chamber as $n=1$ was assessed for the assay in triplicate.

\section{Quantitative Real-time PCR assay}

Following treatment with Elaiophylin, the total RNA was extracted by Trizol reagent according to the manufacturer's instruction. And the total RNA was reverse-transcribed by using HiScript ${ }^{\circledR}$ II Reverse Transcriptase kit. qPCR amplification was conducted using the SYBR Green PCR Master Mix. The thermal profile of reverse transcription was $50^{\circ} \mathrm{C}$ for $15 \mathrm{~min}$ followed by $85^{\circ} \mathrm{C} 5 \mathrm{sec}$. Then cDNA product obtained before was used to qPCR following the thermocycling parameters of $95^{\circ} \mathrm{C}$ for $2 \mathrm{~min}, 40$ amplification cycles of $95^{\circ} \mathrm{C}$ for $5 \mathrm{~s}$, and $60^{\circ} \mathrm{C}$ for $30 \mathrm{~s}$. The relative quantification of the expression levels of the target genes were normalized to $\beta$-ACTIN. The primers of Actin are forwards 5'-CTCTTCCAGCCTTCCTTCCT-3', and reverse, 5'-AGCACTGTGTTGGCGTACAG-3'. The primers of EIF4B are forwards 5'-

AGGGGAAGACTATCTCCCTAACA-3', and reverse, 5'-TCATCCGTTTCATCAGCCCAG-3'. The primers of ITGB1 are forwards 5'-GTAACCAACCGTAGCAAAGGA-3', and reverse, 5'-TCCCCTGATCTTAATCGCAAAAC3'. The primers of ITGA6 are forwards 5'-ATGCACGCGGATCGAGTTT-3', and reverse, 5'TTCCTGCTTCGTATTAACATGCT-3'. The primers of COL4A1 are forwards 5'- 
ACTCTTTTGTGATGCACACCA-3', and reverse, 5'-AAGCTGTAAGCGTTTGCGTA-3'. The expression level of targeted genes were normalized by $\triangle \mathrm{Ct}$ and the relative fold changes were calculated by the $2^{-\triangle \triangle C T}$.

\section{Cell apoptosis detected by flow cytometry assay}

The apoptosis rate of Eca109 and KYSE450 cells was detected by fluorescein isothiocyanate (FITC) Annexin V/propidium iodide (PI) kit according to the manufacturer' instructions. The esophageal squamous carcinoma cells were seeded into $6 \mathrm{~cm}$ dish at a density of $5 \mathrm{X} 105$ cells, and treatment with different concentration of Elaiophylin and transfected with plasmids. Wash the adherent cells with cold PBS, and digested with $0.25 \%$ trypsin without EDTA for $5 \mathrm{~min}$, then resuspended the cell with in medium. After centrifugation, washing the cells twice with cold PBS. Subsequently, resuspended the cells with $100 \mu \mathrm{L}$ binding buffer and added $5 \mu \mathrm{L}$ of Annexin V-FITC and propidium iodide into each tubes. After incubating it $15 \mathrm{~min}$ at room temperature out of light, the samples were detected by flow cytometry within $1 \mathrm{~h}$ by using Cytoflex.

\section{Immunohistochemistry staining of ki67}

The tumor removed from the xenograft were washed in cold phosphate-buffered saline (PBS) and immersed in $4 \%$ paraformaldehyde (PFA) overnight at $4^{\circ} \mathrm{C}$ and dehydration with $20 \%$ sucrose in $1 \times P B S$ for $24 \mathrm{~h}$ at $4^{\circ} \mathrm{C}$. Tissues were embedded in Tissue-Tek ${ }^{\circledR}$ O.C.T. Compound (SAKURA), and cryosections $(16 \mu \mathrm{m})$ were cut onto slides using a Leica CM1950 cryostat. After antigen repair at $100^{\circ} \mathrm{C}$ for $20 \mathrm{~min}$ in critic acid-sodium critrate buffer (PH6.0). After washing with PBS 5 min for three times, blocked with $10 \%$ goat serum for $2 \mathrm{~h}$ after being permeabilized with $0.1 \%$ TrintonX-100 for $10 \mathrm{~min}$, and followed by incubating with primary antibodies overnight at $4{ }^{\circ} \mathrm{C}$. Next day, recycled the primary antibodies and washed the cells with PBS three times. Then incubated the tissues with fluorescein isothiocyanate-labeled secondary antibodies for $1 \mathrm{~h}$ at the room temperature. Whereafter, washed the cells with PBS three times once again and incubated with $A B C T$ (PBS:A:B=50:1:1) for $2 \mathrm{~h}$ at the room temperature. Then washed with PBS and $50 \mathrm{mM}$ Tris. $\mathrm{HCl}$ three times, and developed with $\mathrm{DAB}$ with $1 \mathrm{~min}$, and washed with $\mathrm{dH}_{2} \mathrm{O}$ once again. Finally, dehydration with $\mathrm{C} 2 \mathrm{H} 5 \mathrm{OH}$ and vitrification with xylene, sealed the slides with neutral resins. Roasted at $50^{\circ} \mathrm{C}$ overnight.

\section{Xenograft mice model}

The male BALB/C-Nu mice (5-7 weeks old) was brought from HUNAN SJA LABORATORY ANIMAL CO., LTD. (Hunan China), all animal experiments were performed in strict accordance with the guidelines of the Research Ethics Committee of Wuhan university. All experimental protocols were approved by Ethics Committee of Wuhan University. Number: MLICJ2021013. Approval Number: MRI2021-LACJ013. Eca109 cells $\left(5 \times 10^{6}\right)$ resuspended in $200 \mu \mathrm{L}$ of PBS were injected subcutaneously into the right flanks of nude mice. After two weeks, Elaiophylin was given i.p. $(1 \mathrm{mg} / \mathrm{kg})$ every two days for 21 days, or DMSO was 
used as a control. Finally, the xenograft tumors were removed and weighted. Tumor volumes were calculated as length*(square of width)/2. The study is reported in accordance with ARRIVE guidelines. For euthanasia, the mice were intravenous injected with barbiturate for $150 \mathrm{mg} / \mathrm{kg}$.

\section{Statistical analysis}

All experiments were performed at least three times. All data was presented as means \pm SEM. $P$ value $<0.05$ was considered significantly different. Statistical comparisons between two groups were made using Student's t-test. Statistical analysis and graphs were produced using GraphPad Prism 8 software.

\section{Results}

\section{Elaiophylin is a late-stage autophagy inhibitor in the ESCC cells}

To identify whether Elaiophylin play as an autophagy inhibitor like it does in ovarian cancer and multiple myeloma with mutant TP53, we treated ESCC cells with different concentrations, with CQ as a positive control. The western blot assay results indicated that the ratio of LC3II/LC3I was increased after exposed to Elaiophylin. And the expression level of p62 was upregulated at the protein level in the Eca109 (Figure1. A) and KYSE450 cells (Figure1. B). We further transfected GFP-LC3 fusion protein to ESCC cells, then exposed to Elaiophylin with different concentrations. The results showed that the GFP-LC3 puncta was increased in a dose-dependent manner after Elaiophylin treated in the Eca109 and KYSE450 cells respectively (Figure1. C, E). The statistical analysis of C and E (Figure1. D, F). Then, when exposed Elaiophylin combined with CQ, the accumulated LC3-II and p62 by Elaiophylin was not further increased by CQ, which indicated that Elaiophylin blocked autophagic flux similar to CQ (Figure1. G). To further verify whether Elaiophylin can inhibit the autophagy at the late stage, we transfected mCherry-GFP-LC3 plasmid to ESCC cells. Since EGFP fluorescence is quenched by acid protease in the lysosome, thus the puncta of yellow was represented of autophagosome, whereas the red was represented of autolysosome (Figure1. H, I). Statistical analysis of G (Figure1. J). The results indicated that Elaiophylin could inhibit autophagy flux at the late stage in the ESCC cells.

\section{Elaiophylin exerts anti-proliferative, anti-migrative, and pro-apoptotic effects on ESCC cells}

To assess the other potential function of Elaiophylin on ESCC cells, we detected the cell viability, proliferation, migration and apoptosis rate of the cells and confirmed its effect by measuring the protein expression level of E-cadherin, Bax and Bcl-2 after dealing with Elaiophylin. Utilizing the CCK8 assay, we observed that the cell viability was decreased by Elaiophylin in a dose-dependent manner in both two ESCC cell lines when compared with the control. (Figure2. A, B). As cancer metastasis contributed to poor prognosis of ESCC patients, transwell migration assay was also carried out to determine the effects of 
Elaiophylin on the ability of migration of ESCC cells. After staining with crystal violet, the inhibition of ESCC cell migration was evident, as less cell numbers were observed after treating with Elaiophylin compared to the control. (Figure2. C). Statistical analysis results of C (Figure2. D). In addition, the results of immunofluorescent staining for Ki67 showed that the Ki67 positive cells was decreased by Elaiophylin, which indicated that the proliferation rate of ESCC cells was attenuated (Figure2. E, G). Statistical analysis results of E, G (Figure2. F, H). Notably, flow cytometry assay established increased apoptosis rates in ESCC cells by treating with Elaiophylin. At the same time, the normal esophagus cell lines HET-1A showed a lower toxicity response to Elaiophylin. (Figure2. I). Statistical analysis results of I (Figure2. J). Moreover, western blot assay analysis showed increased protein expression of E-cadherin, Bax by Elaiophylin, whereas the Bcl-2 expression level was decreased (Figure2. K, L, M).

\section{RNA-seq results indicated that Elaiophylin could inhibit the activation of PI3K/AKT signaling pathway}

To elucidate the mechanism and target of Elaiophylin, we conducted RNA-seq analysis (GSE171167) to identify the genes that were transcriptionally affected by Elaiophylin. In RNA-seq, the correlation of expression levels between samples is an important indicator to test the reliability of the experiment and whether the sample selection is reasonable. The closer the correlation coefficient is to 1 , the higher the similarity of the expression patterns between samples. The Encode plan recommends that the square of Pearson's correlation coefficients of the two biological replicates in each group of the two cell lines are both greater than 0.97 , indicating that the sample has good reproducibility and the sequencing results are credible (Figure3. A, F). Then the differently expressed genes were screened, and the screening criterion was $\mid \log 2$ (FoldChange) $\mid>1 \&$ padj $<0.05$. The volcano chart shows the distribution of differential genes for each comparison combination (Figure3. B, G). Red dots indicate genes that are up-regulated, and green dots indicate genes that are down-regulated. The results showed that there were 360 up-regulated genes and 891 down-regulated genes in Eca109 cells (Figure3. B). And there are 126 up-regulated genes and 435 down-regulated genes in KYSE450 cells (Figure3. G). Then cluster all differentially expressed genes. The differential genes of all comparison groups are combined as the differential gene set. We use mainstream hierarchical clustering to perform cluster analysis on the FPKM value of genes, and perform uniform processing on the rows. Shown in the form of a heat map. Genes with similar expression patterns in the heat map will be grouped together. The color in each grid is the value obtained after the row of expression data is normalized. Therefore, the colors in the heat map can only be compared horizontally, not vertically. The results showed that in the two cell lines, the expression levels of differentially expressed genes in different samples were significantly different (Figure3. C, H). Finally, we used clusterProfiler software to perform GO (Gene Oncology) function enrichment analysis for differential genes, and KEGG (Kyoto Encyclopedia of Genes and Genomics) function enrichment analysis. GO is a comprehensive database describing gene function, divided into three parts: biological process and cell composition, and molecular function. We mainly focus on the enrichment analysis of biological processes. Figure 3D mainly shows the results of the enrichment analysis of the biological process of Eca109 cells. The 
abscissa in the figure represents the ratio of the number of differential genes annotated to GO Term to the total number of differential genes, the ordinate is GO Term, and the size of dot represents the annotation to $\mathrm{GO}$ the number of genes on Term, the color from red to purple represents the significance of enrichment. The results showed that in the two kinds of cells, Elaiophylin mainly affected the binding of intercellular cadherin molecules and intercellular adhesion molecules (Figure3. D, I). KEGG enrichment results show that the abscissa is the ratio of the number of differential genes, the ordinate is the KEGG pathway, the size of the dot represents the number of genes annotated to the KEGG pathway, and the color from red to purple represents enrichment. The results show that Elaiophylin mainly affects the PI3KAkt signaling pathway in the two cell lines (Figure3. E, J).

\section{Elaiophylin regulates ESCC cells through regulating proteins related to PI3K/AKT signaling pathway}

For further exploring the mechanism by which Elaiophylin regulated cell growth and cell apoptosis, a human cancer pathway phosphorylation antibody array was used to detect the pression and phosphorylation of the proteins related to the cancer signaling pathway. 93 proteins and its phosphorylation sites which involved in MAPK signaling pathway, Apoptosis signaling pathway, AKT signaling pathway, Cell cycle, Jak-STAT signaling pathway, p53 signaling pathway, Regulation of actin cytoskeleton, Wnt signaling pathway, VEGF signaling pathway, NF-KB signaling pathway, mTOR signaling pathway and TGF-beta signaling pathway were examined, among which downregulated were annotated to KEGG analysis, the results showed that the downregulated proteins and its phosphorylation sites were mainly enrichment in the MAPK and PI3K/AKT signaling pathway, which indicated that the PI3K/AKT pathway was inhibited (Figure4. A, C). As shown in figure 4B, D, some proteins and its phosphorylation sites were exhibited significantly downregulated levels, among which JAK2, PP2A-alpha, 14-3-3 zeta/delta, HER2, IKK alpha/beta, ATF2, p53, EGFR and Myc (Phospho-Ser62), JAK2 (Phospho-Tyr1007), 4E-BP1 (Phospho-Thr45) were included. Then, to analyze the genes that were downregulated by Elaiophylin both in Eca109 and KYSE450 cells, we firstly found the common DEGs by Venn (Figure4. E). Then we annotated the downregulation DEGs to KEGG analysis, as predicted, the results showed that the genes were mostly enrichment in the PI3K-Akt signaling pathway (Figure4. F). Hence, we verified the genes enrichment in PI3K-Akt pathway by RT-qPCR assay. Noteworthy is that the expression of COL4A1, EIF4B, ITGA5 and ITGB1 was blocked at the mRNA level both in the Eca109 and KYSE450 cells (Figure4. $\mathrm{G)}$.

\section{Elaiophylin might suppress the expression of EIF4B via PI3K/AKT signaling pathway}

Since EIF4B was previously reported to play an essential role in cell proliferation and survival, especially in cancer cells, but has not been explored in esophageal cancer, we therefore checked the EIF4B protein expression in the Elaiophylin treated cells. Furthermore, we also assessed the PI3K, p-PI3K, AKT, p-AKT 
protein expression level. Finally, we observed that the EIF4B, p-PI3K, AKT, p-AKT protein expression level was downregulated by Elaiophylin, which further confirmed that the activity of PI3K-AKT signaling pathway was inhibited (Figure5. A). When exposed to LY294002, the PI3K inhibitor, the expression of EIF4B was downregulated both in Eca109 and KYSE450 cells (Figure5. B). And, when autophagy was blocked by CQ and shATG5 and ATG7, the expression of EIF4B was impaired as well (Figure5. C, D). In order to investigate whether Elaiophylin induces apoptosis via PI3K/AKT pathway, a vector expressing AKT (T308D/S473D), the constitutively active form of AKT, was transfected into Eca109 and KYSE450 cells and the p-mTOR was induced (Figure5. E). The results showed that the ectopic expression of AKT (T308D/S473D) significantly increased the proliferation rate of Elaiophylin-treated Eca109 and KYSE450 cells (Figure5. F, G). In addition, the Elaiophylin-induced apoptosis was abrogated by AKT (T308D/S473D) overexpression (Figure5. H, I). Taken together, from what discussed before, we speculated that Elaiophylin might target to EIF4B to regulate the proliferation, migration and apoptosis of ESCC cells via PI3K/AKT signaling pathway.

\section{Knockdown of EIF4B suppresses the proliferation, migration of ESCC cells and promotes apoptosis}

To further confirm the function of EIF4B in ESCC cells, we continue knocked down the EIF4B in ESCC cells to test if silencing EIF4B affect its proliferation, migration and apoptosis. All cells were divided into three groups, scramble, shEIF4B and shEIF4B+EIF4B-OE. First of all, western blot assay was employed to detect the EIF4B expression across all groups, the results demonstrated that the EIF4B was effectively knocked down after transfection of shEIF4B, and was reversed by co-transfection with EIF4B-OE (Figure6. A, B). Transwell assay showed that the migratory ability was attenuated when silencing the EIF4B, and can be reversed by co-transfection with EIF4B-OE (Figure6. C, D). And efficient shRNA-directed knockdown of EIF4B expression could inhibit the proliferation of Eca109 and KYSE450 cells, as the percentage of ki67 positive cells was decreased in the shEIF4B group compared to scramble and shEIF4B+EIF4B-OE group (Figure6. E, F, G, H). Using Annexin V-FITC/PI assay, we also observed that effective knockdown of EIF4B could accelerate the apoptosis of ESCC cells compared to the scramble and shEIF4B+EIF4B-OE group (Figure6. I, J). Additionally, western blot assay examined the protein expression of E-cadherin, Bax, and $\mathrm{Bcl}-2$, the results showed that E-cadherin, Bax was upregulated when silencing EIF4B and Bcl-2 was downregulated, which was reversed by overexpression of EIF4B (Figure6. K, L). All the data suggested that knockdown of EIF4B could inhibit the proliferation and migration of ESCC cells and promotes its apoptosis. Whereas, overexpression of EIF4B on the basis of blocking the expression of EIF4B abrogated the effect of silencing of EIF4B, which indicated that the EIF4B might play an important role in ESCC progression.

\section{Upregulation of EIF4B could rescued the phenotype on ESCC cells caused by Elaiophylin}


To ascertain whether the Elaiophylin affects the ability of proliferation, migration and apoptosis of ESCC cells via EIF4B, we transfected elF4B-OE plasmid to ESCC cells on the basis of treating with Elaiophylin. All cells were divided to three groups vector, Elaiophylin only and EIF4B-OE on the basis of Elaiophylin. Firstly, the EIF4B protein expression level was examined by western blot, the result indicated that it was down regulated by Elaiophylin, but was reversed by co-transfection of EIF4B-OE. (Figure7. A). And, Elaiophylin induced accumulated LC3-II and p62 were both recovered in EIF4B-overexpressing Eca109 and KYSE450 cells (Figure7. B). The transwell migration assay showed that transfection with EIF4B-OE could blocked the migration-inhibitory effect resulted from Elaiophylin (Figure7. C, D). Interestingly, the IHC of ki67 of the different groups illustrated that the transfection with EIF4B-OE on the basis of Elaiophylin could enhance the proliferation of Eca109 and KYSE450 cells when compared with the Elaiophylin only group. (Figure7. E, F, G, H). In addition, the cell apoptosis rate was also decreased when transfection with EIF4B-OE on the basis of Elaiophylin compared to the Elaiophylin only group as detected by flow cytometry (Figure6. I, J). In the end, in order to confirm the effect of different groups on the proliferation, migration and apoptosis in Eca109 and KYSE450 cells, the protein level of E-cadherin, Bax, Bcl-2 was examined by western blot analysis. The result showed that the expression level of E-cadherin, Bax, Bcl-2 in the Elaiophylin group was reversed by transfection of EIF4B-OE (Figure7. K, L). The phenomenon indicated that elF4B-OE could reverse the effect of the Elaiophylin on ESCC cells.

\section{Elaiophylin inhibit the tumor growth of esophageal cancer in vivo}

As Elaiophylin could inhibit the proliferation of ESCC cells in the vitro, we further investigated the effect of Elaiophylin on tumor growth in vivo. The Eca109 cell line was used to inject into the right flanks of nude mice to construct an in vivo model. After two weeks, Elaiophylin was given i.p. $(1 \mathrm{mg} / \mathrm{kg})$ every two days for 21 days, or DMSO was used as a control. The tumor was measured every 7 days, the results showed the volume of the tumors in the Elaiophyin group was significantly decreased than the control group (Figure8. B). Finally, the tumors were taken from the mice, weighted and photographed followed by measurement of the volume (Figure8. A, C, D). We found that the Elaiophylin group was lighter and smaller than the control group. The IHC staining of Ki67 showed that the expression of Ki67 was obviously reduced in the Elaiophylin group when compared with the control (Figure8. E). In a word, these results suggest that Elaiophylin could inhibit the progression of esophageal tumor in vivo.

\section{Discussion}

Previous studies have reported that Elaiophylin was a novel autophagy inhibitor and showed potent antitumor activity in many kinds of tumors. For instance, Elaiohylin exerted prominent antitumor efficacy as an autophagy inhibitor in ovarian cancer ${ }^{7}$ and multiple myeloma with mutant TP53, which was also involved ER stress-associated apoptosis ${ }^{8}$. Besides, Elaiophylin could inhibit tumor progression and suppressed tumor growth in xenograft of metastatic castration-resistant prostate cancer (mCRPC), which 
was targeting the orphan nuclear receptor RORy ${ }^{13}$. On the other hand, Elaiophylin has also been proved to inhibit angiogenesis in cancers as well as proliferation, migration and invasion accompanied by it. However, little is known about the function of Elaiophylin in ESCC. Our findings that Elaiophylin inhibits the autophagy at the late stage is in line with the previous reports reported in ovarian cancer ${ }^{7}$ as well as multiple myeloma with mutant TP5 $3^{8}$. In addition, our results showed that Elaiophylin could inhibit the proliferation and migration of ESCC cells and promotes its apoptosis.

The eukaryotic initiation factor 4B (EIF4B) phosphorylation can be stimulated by PI3K/AKT/mTOR signaling pathways ${ }^{11}$, which is on Ser $422^{14}$. In relation to this, we identified that the EIF4B was inhibited by the suppression of PI3K/AKT signaling pathway in ESCC cells. An increasing number of studies have also indicated that EIF4B is necessary for cell proliferation and survival, and its effect is achieved by regulating the translation of proliferation and survival-related mRNA ${ }^{15}$. And EIF4B can play an important anti-apoptotic effect in acute leukemia cells by regulating the expression of anti-apoptotic proteins Bcl-2 and Bcl-xl. According to the relevant studies, knockdown of EIF4B can reduce the rate of cell proliferation, promote caspase-dependent apoptosis, and make cells more sensitive to camptothecin-induced cell death. EIF4B can inhibit the apoptosis of acute leukemia cells ${ }^{11}$. And EIF4B can promote the proliferation and metastasis of hepatocellular carcinoma ${ }^{12}$. MiR-216a can inhibit the development of oral squamous cell carcinoma ${ }^{16}$ and non-small cell lung cancer ${ }^{17}$ by targeting EIF4B. Intriguingly, it was recently reported that EIF4B was also shown to be a novel marker for poor prognosis of diffuse large B-cell lymphoma ${ }^{18}$. All of the results indicated that EIF4B is closely associated with the occurrence and progression of a various of tumors. According to the human cancer pathway phosphorylation antibody array and RNA-seq analysis, when dealing the ESCC cells with Elaiophylin the activity of PI3K/AKT signaling pathway was negatively regulated. At the same time, the expression of EIF4B was also downregulated at both the mRNA and protein level via the PI3K/AKT signaling pathway.

In addition, given that EIF4B can regulate the proliferation and survival of many kinds of cancers. We further knockdown the EIF4B of ESCC cells, which contributed to the lower proliferation and migration and higher apoptosis rate. Meanwhile, in combination with a EIF4B-OE vector on the basis of silencing of EIF4B, the effect of downregulation of EIF4B on ESCC cells is offset, which indicated that silencing EIF4B could inhibit the proliferation and migration of ESCC cells and promote apoptosis. Of note, forced EIF4B expression blocked the effect of Elaiophylin and had an effective promotion ability of proliferation and migration and inhibition of apoptosis of ESCC cells. The results of our study showed that EIF4B might be a target of Elaiophylin to regulate the proliferation, migration and apoptosis of ESCC cells. To further verify our results, we conducted the experiments in vivo. The results showed that Elaiphylin could meanwhile inhibit the tumor growth of esophageal cancer in vivo.

In summary, in this study we firstly found that Elaiophylin could serve as an autophagy inhibitor in ESCC cells. In addition, our results indicated that knockdown of EIF4B could inhibit the proliferation, migration of ESCC cells and promote apoptosis, which was could blocked by EIF4B-OE. Then we revealed that EIF4B has an opposite effect of Elaiophylin and EIF4B-OE further rescued the effect of Elaiophylin on ESCC 
cells, which indicated that Elaiophylin could meanwhile target EIF4B via PI3K/AKT signaling pathway to inhibit the viability, proliferation and migration of ESCC cells and to promote its apoptosis. Moreover, Elaiophylin could inhibit the growth of esophageal tumor in vivo. In conclusion, our study identified that Elaiophylin may serve as a new therapeutic medicine for ESCC patients, and improve patient outcomes.

\section{Conclusions}

Together, our results indicated that Elaiophylin is an autophagy inhibitor governing growth and migratory ability of ESCC cells through PI3K/AKT signaling pathway and inhibit the proliferation of esophageal tumor in vivo. simultaneously, and identified EIF4B as a target of Elaiophylin, which might provide a new therapeutic strategy for esophageal squamous cell carcinoma.

\section{Declarations}

Ethics approval and consent to participate: All animal experiments were performed in strict accordance with the guidelines of the Research Ethics Committee of Wuhan university. All experimental protocols were approved by Ethics Committee of Wuhan University. Number: MLICJ2021013.

Consent for publication: All authors have read this manuscript and would like to have it considered exclusively for publication in Journal of Cancer Cell International.

Availability of data and materials: The datasets generated during the current study are available in the GEO repository, GSE116717.

Competing interests: None.

Funding: National Natural Science Foundation of China (No. U1604175).

Authors' contributions: Chen Yongshun have made substantial contributions to the conception, Gao Lijuan contributes to the design of the work and drafted the work and substantively revised it. LiBin contributes to the creation of new software used in the work. All authors read and approved the final manuscript.

Acknowledgements: None.

\section{References}

1. Domper Arnal, M. J., Ferrández Arenas, Á. \& Lanas Arbeloa, Á. Esophageal cancer: Risk factors, screening and endoscopic treatment in Western and Eastern countries. World J Gastroentero/ 21, 7933-7943, doi:10.3748/wjg.v21.i26.7933 (2015).

2. Short, M. W., Burgers, K. G. \& Fry, V. T. Esophageal Cancer. Am Fam Physician 95, $22-28$ (2017). 
3. Lin, Y. et al. Esophageal cancer in high-risk areas of China: research progress and challenges. Ann Epidemiol 27, 215-221, doi:10.1016/j.annepidem.2016.11.004 (2017).

4. Wang, M., Smith, J. S. \& Wei, W. Q. Tissue protein biomarker candidates to predict progression of esophageal squamous cell carcinoma and precancerous lesions. Ann N Y Acad Sci 1434, 59-69, doi:10.1111/nyas.13863 (2018).

5. Hirano, H. \& Kato, K. Systemic treatment of advanced esophageal squamous cell carcinoma: chemotherapy, molecular-targeting therapy and immunotherapy. Jpn J Clin Oncol 49, 412-420, doi:10.1093/jjco/hyz034 (2019).

6. Lam, A. K. Introduction: Esophageal Squamous Cell Carcinoma-Current Status and Future Advances. Methods Mol Bio/ 2129, 1-6, doi:10.1007/978-1-0716-0377-2_1 (2020).

7. Zhao, X. et al. Elaiophylin, a novel autophagy inhibitor, exerts antitumor activity as a single agent in ovarian cancer cells. Autophagy 11, 1849-1863, doi:10.1080/15548627.2015.1017185 (2015).

8. Wang, G. et al. The novel autophagy inhibitor elaiophylin exerts antitumor activity against multiple myeloma with mutant TP53 in part through endoplasmic reticulum stress-induced apoptosis. Cancer Biol Ther 18, 584-595, doi:10.1080/15384047.2017.1345386 (2017).

9. Andreou, A. Z. \& Klostermeier, D. elF4B and elF4G jointly stimulate elF4A ATPase and unwinding activities by modulation of the elF4A conformational cycle. J Mol Biol 426, 51-61, doi:10.1016/j.jmb.2013.09.027 (2014).

10. Andreou, A. Z., Harms, U. \& Klostermeier, D. Single-stranded regions modulate conformational dynamics and ATPase activity of elF4A to optimize 5'-UTR unwinding. Nucleic Acids Res 47, 52605275, doi:10.1093/nar/gkz254 (2019).

11. Ma, Y. et al. [Synergistic role of JAK/STAT5 and PI3K/AKT signaling pathways in regulating elF4B in acute leukemia]. Sheng Wu Gong Cheng Xue Bao 36, 2413-2423, doi:10.13345/j.cjb.200015 (2020).

12. Xu, J. et al. Long noncoding RNA GMAN promotes hepatocellular carcinoma progression by interacting with elF4B. Cancer Lett 473, 1-12, doi:10.1016/j.canlet.2019.12.032 (2020).

13. Zheng, J. et al. Targeting castration-resistant prostate cancer with a novel RORy antagonist elaiophylin. Acta Pharm Sin B 10, 2313-2322, doi:10.1016/j.apsb.2020.07.001 (2020).

14. Bettegazzi, B. et al. elF4B phosphorylation at Ser504 links synaptic activity with protein translation in physiology and pathology. Sci Rep 7, 10563, doi:10.1038/s41598-017-11096-1 (2017).

15. Shahbazian, D., Parsyan, A., Petroulakis, E., Hershey, J. \& Sonenberg, N. elF4B controls survival and proliferation and is regulated by proto-oncogenic signaling pathways. Cell Cycle 9, 4106-4109, doi:10.4161/cc.9.20.13630 (2010).

16. Li, L. \& Ma, H. Q. MicroRNA-216a inhibits the growth and metastasis of oral squamous cell carcinoma by targeting eukaryotic translation initiation factor 4B. Mol Med Rep 12, 3156-3162, doi:10.3892/mmr.2015.3761 (2015).

17. Wang, R. T., Xu, M., Xu, C. X., Song, Z. G. \& Jin, H. Decreased expression of miR216a contributes to non-small-cell lung cancer progression. Clin Cancer Res 20, 4705-4716, doi:10.1158/1078-0432.Ccr14-0517 (2014). 
18. Horvilleur, E. et al. A role for eukaryotic initiation factor 4B overexpression in the pathogenesis of diffuse large B-cell lymphoma. Leukemia28, 1092-1102, doi:10.1038/leu.2013.295 (2014).

\section{Figures}

A

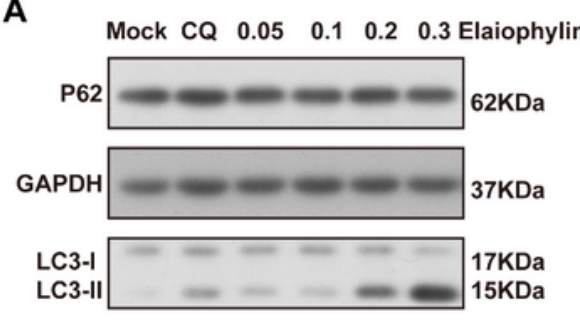

B

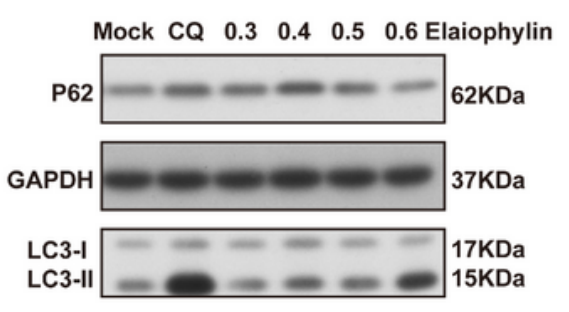

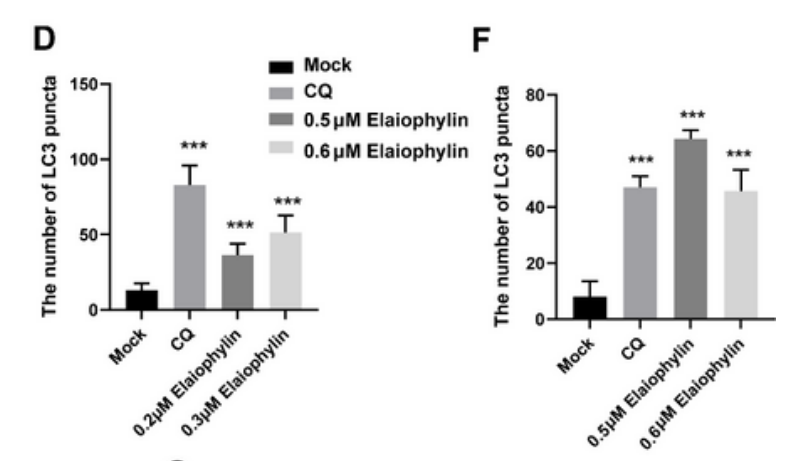

E
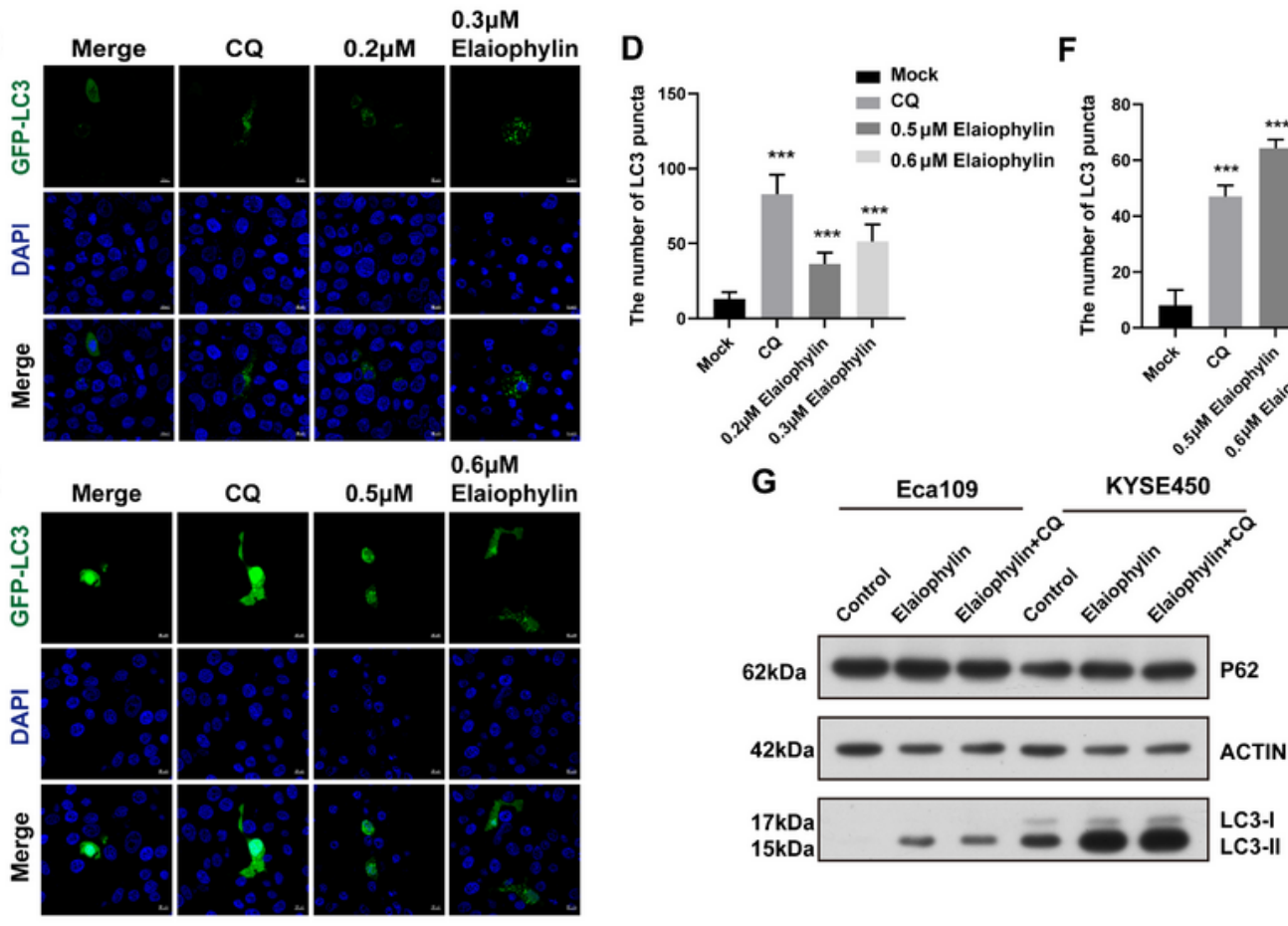

H

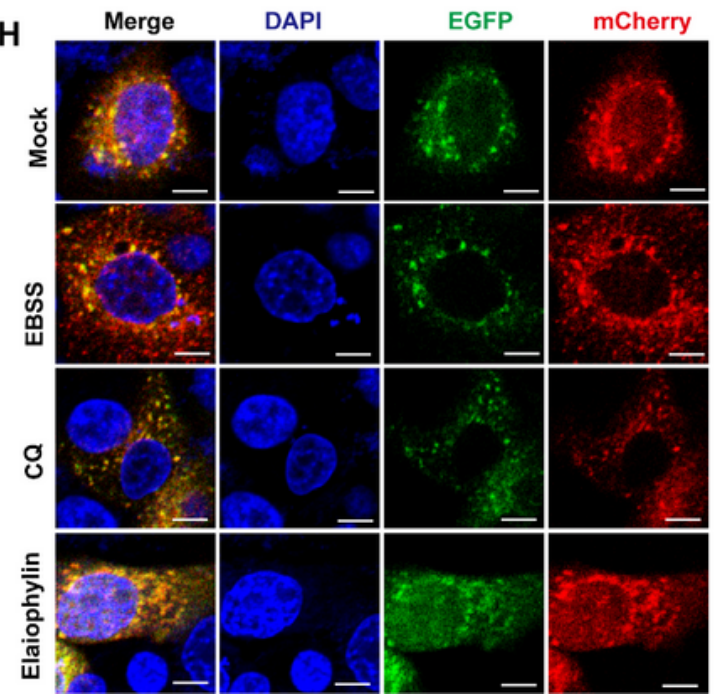

I

mCherry-GFP-LC3

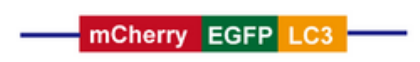

J $\square$ Autophagosome Autolysosome

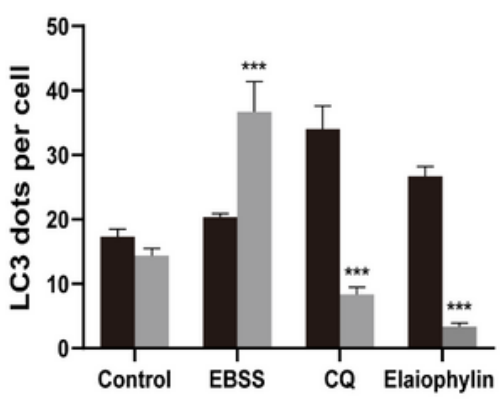

Figure 1 
Elaiophylin could inhibit autophagy flux at the late stage in the ESCC cells. (A, B) Western Blot assay was carried out to detect the level of LC3 and p62 protein in Eca109 and KYSE450 following treatment with different concentrations of Elaiophylin. The grouping of gels cropped from different parts of the same gel and the same exposures. (C, E) The LC3 puncta in Eca109 and KYSE450 cells was visualized by confocal microscope after transfected with GFP-LC3 fusion protein. (D, F) Statistical analysis of C, E. Error bars represent the mean $\pm S E M$. (G) Western Blot assay was carried out to detect the level of LC3 and p62 protein in Eca109 and KYSE450 following treatment with different concentrations of Elaiophylin in combination with $\mathrm{CQ}$. The grouping of gels cropped from different parts of the same gel and the same exposures. $(\mathrm{H})$ After transfected with mCherry-EGFP-LC3 plasmids followed by treatment with Elaiophylin, the puncta of LC3 were visualized by confocal microscope, the scale bar was $10 \mu \mathrm{m}$. The puncta of yellow are represented of autophagosome, whereas the red was represented of autolysosome. (I) The structure of the mCherry-EGFP-LC3 plasmids. (J) Statistical analysis of autolysosome compared with the autophagosome. Error bars represent the mean \pm SEM. Statistical analysis was generated from unpaired ttest, asterisks denote statistical significance as $\star \star \star p \otimes 0.001$. 

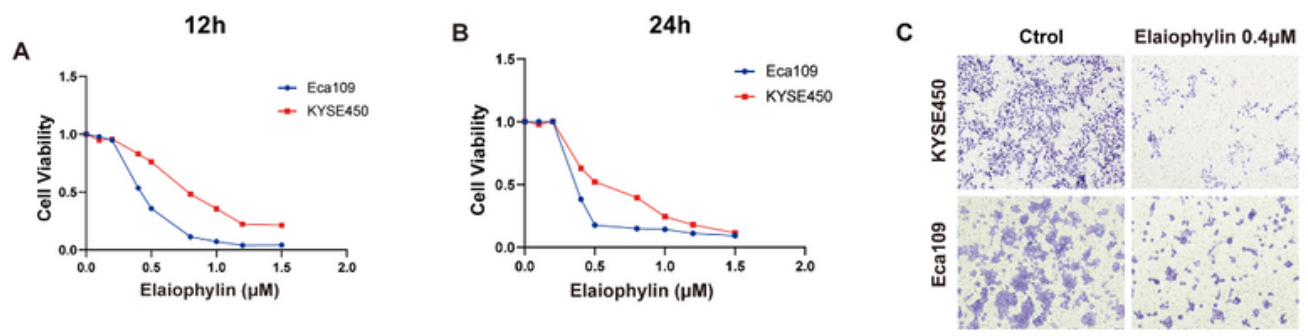

D
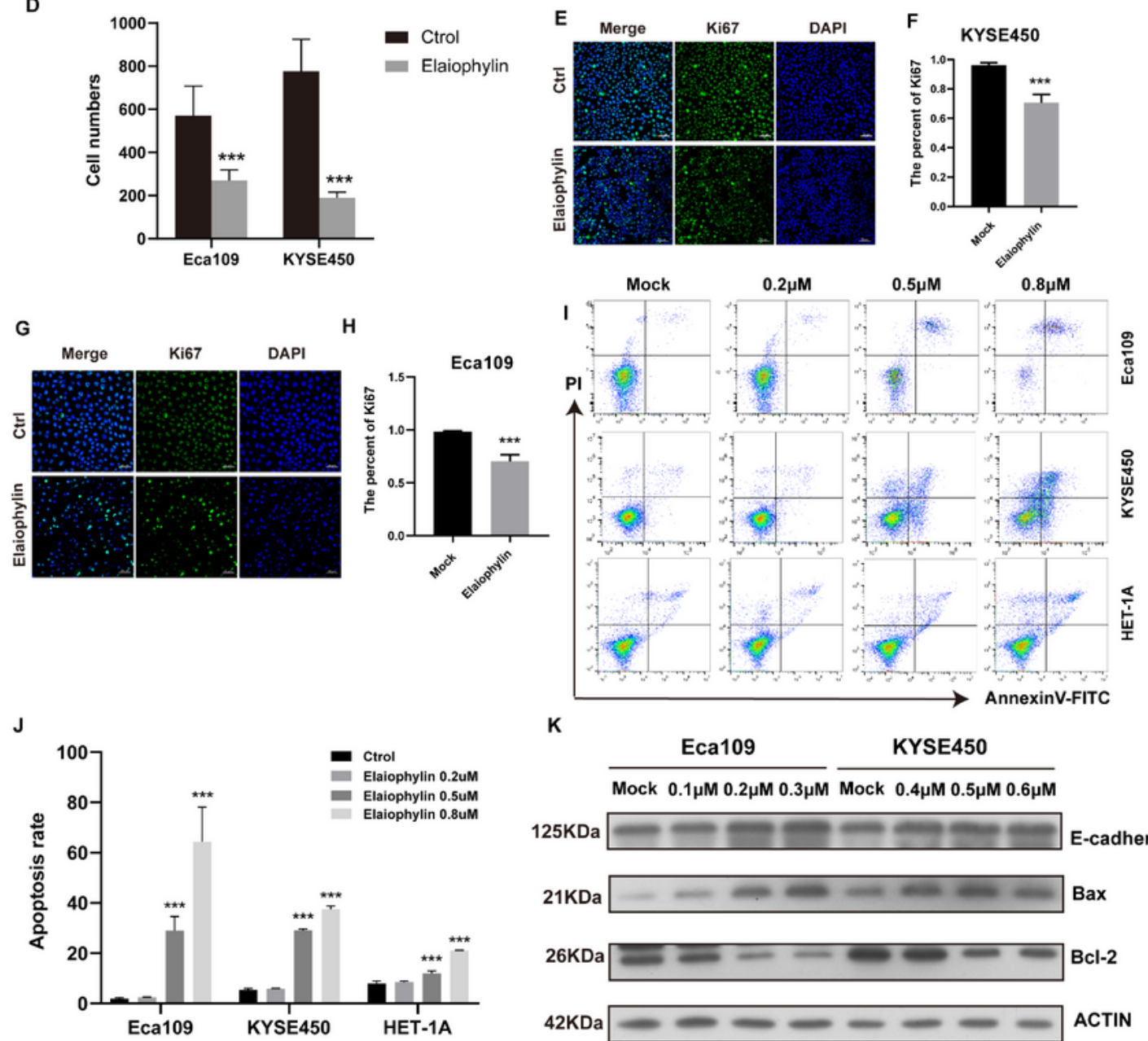

K

L

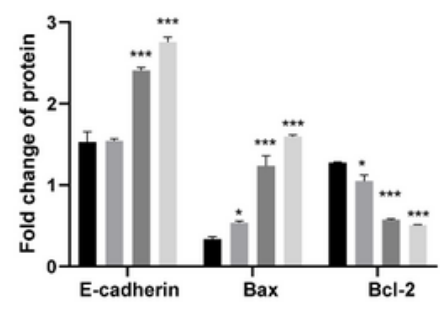

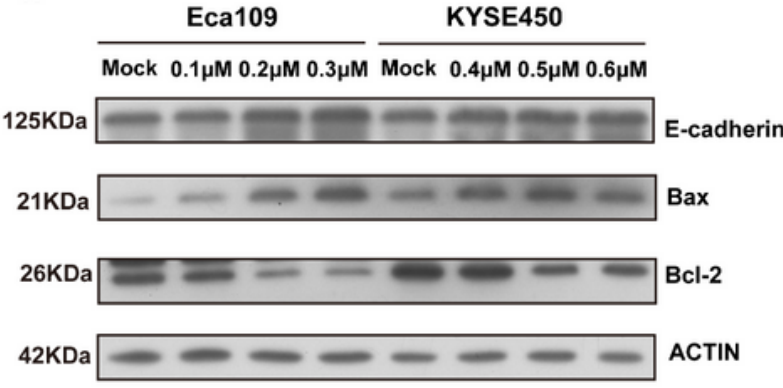

M

KYSE450

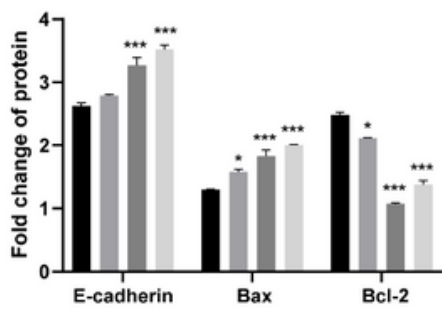

- Mock

$0.2 \mu \mathrm{M}$ Elaiophylin

- $0.5 \mu \mathrm{M}$ Elaiophylin $0.8 \mu \mathrm{M}$ Elaiophylin

Figure 2

Elaiophylin exerts anti-proliferative, anti-migrative, and pro-apoptotic effects on ESCC cells. (A, B) The CCK-8 assay was utilized to explore the effect of Elaiophylin on the proliferation of Eca109 and KYSE450 cells. (C) The migration ability between the control and Elaiophylin group was detected by transwell assay. (D) Statistical analysis of the cell numbers migrated to the other side of the chamber. Error bars represent the mean \pm SEM. $(E, G) I H C$ of ki67 was used to detect the proliferation rate of Eca109 and 
KYSE450 cells following by treatment with Elaiophylin. $(F, H)$ Statistical analysis result of the IHC showed that the ki67 expression level was downregulated in the Elaiophylin group compared with the control. Error bars represent the mean \pm SEM. (I) The apoptosis rate was measured by Annexin V/PI in Eca109 and KYSE450 cells. Cells with Annexin V-FITC positive and PI negative or both Annexin V-FITC and PI positive were considered apoptotic. $(\mathrm{J})$ Statistical analysis of the apoptosis rate of the two cells. Error bars represent the mean \pm SEM. (K) The protein level of E-cadherin, Bax, Bcl-2 was measured in Eca109 and KYSE450 cells after treatment with different concentrations of Elaiophylin. The E-Cadherin, Bax, Actin was cropped from different parts of the same gel, and Bcl-2 was from different gels and from the same exposures. (L, M) Statistical analysis of the protein expression in K. Error bars represent the mean $\pm S E M$. Statistical analysis was generated from unpaired t-test, asterisks denote statistical significance as *p》 0.05 , *** $\mathrm{p} \varangle 0.001$. 


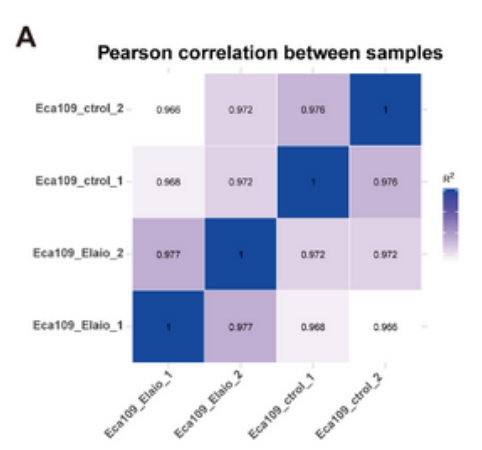

D

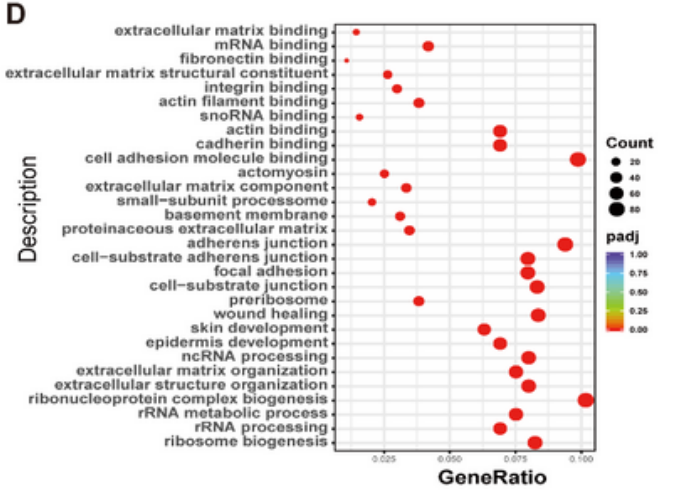

F
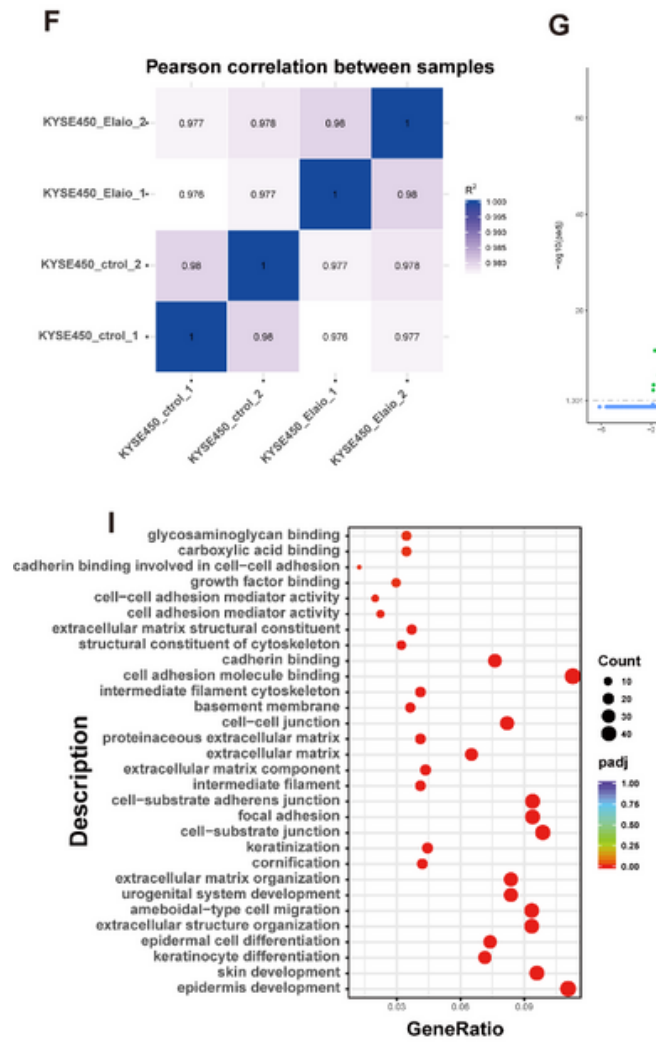

G

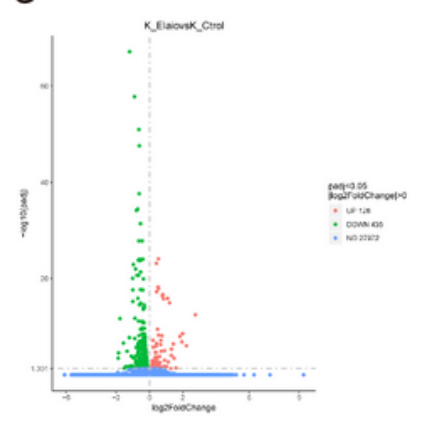

C
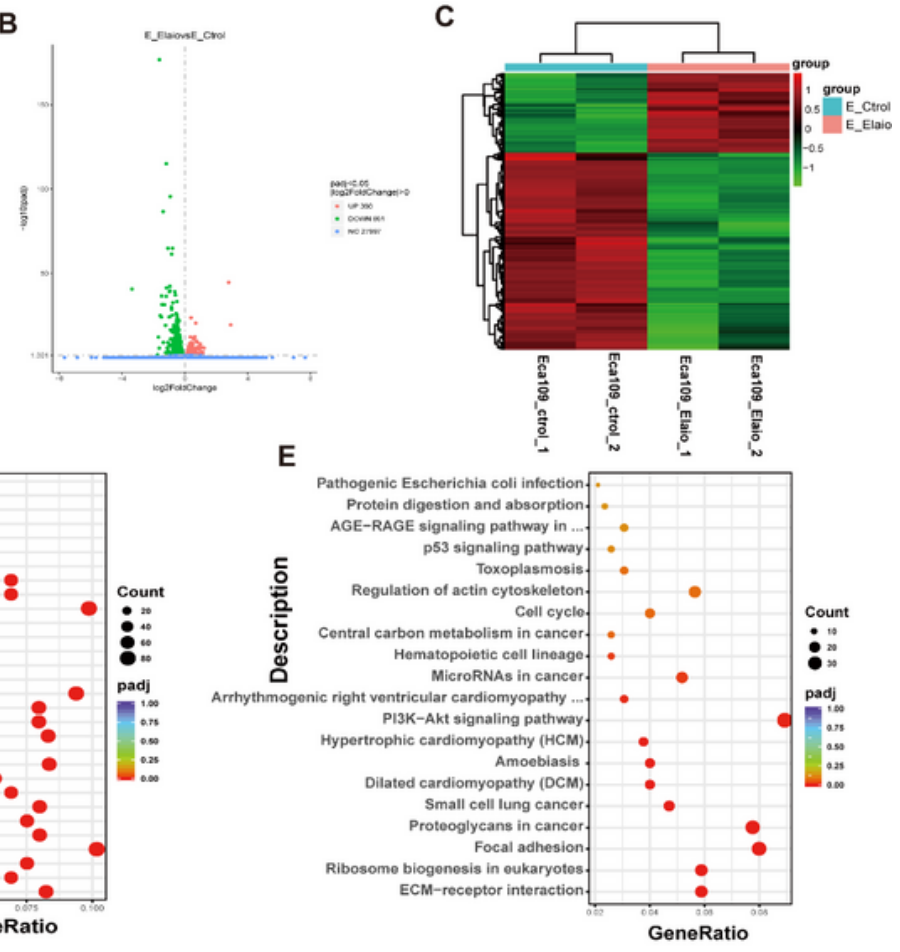

H

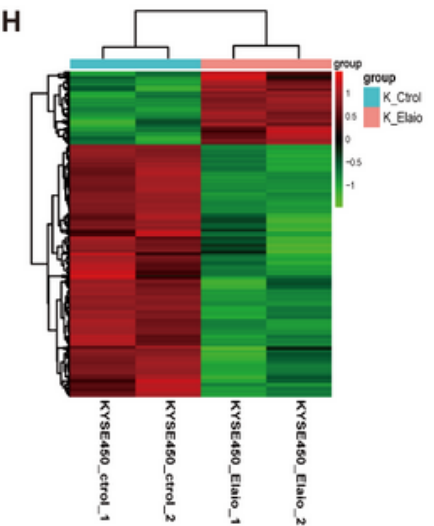

$J$
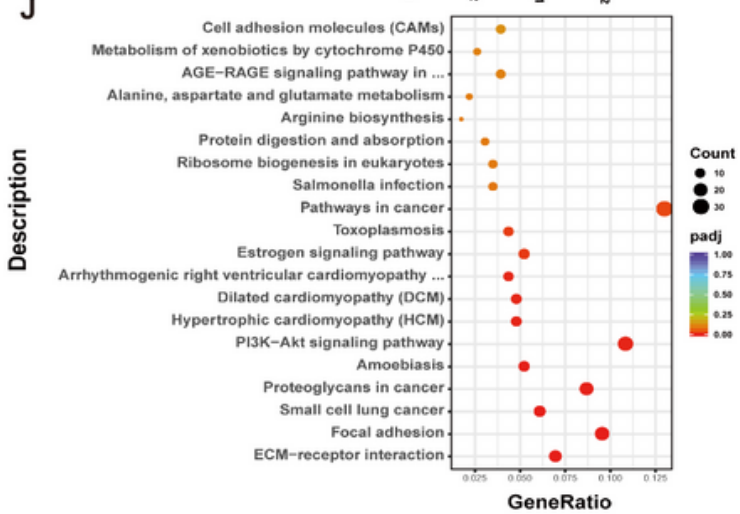

Estrogen signaling pathway
ventricular cardiomyopathy Dilated cardiomyopathy (DCM). Hypertrophic cardiomyopathy (HCM).

PI3K-Akt signaling pathway Proteoglycans in cancer. Small cell lung cancer. ECM-receptor interaction

\section{Figure 3}

RNA-seq analysis of the Eca109 and KYSE450 cells after treatment of Elaiophylin for 12h. (A, F) Heat map showing the hierarchically clustered Pearson correlation matrix resulted from comparing the transcript expression values of the control and Elaiophylin samples in Eca109 and KYSE450 cells respectively. $(B, G)$ Identification of Elaiophylin regulated genes in Eca109 and KYSE450 cells. Upregulated genes are labelled in red, whereas downregulated genes are labelled in blue in the volcano 
plot. (C, H) Hierachical clustering of DEGs in control and Elaiophylin samples in Eca109 and KYSE450 cells. FPKM values are log2-transformed and then median-centred by each gene. (D, I) The top 30 representative GO biological processes of Elaiophylin downregulated genes in Eca109 and KYSE450 cells. $(E, J)$ The top 30 representative KEGG pathways of Elaiophylin regulated genes in Eca109 and KYSE450 cells.

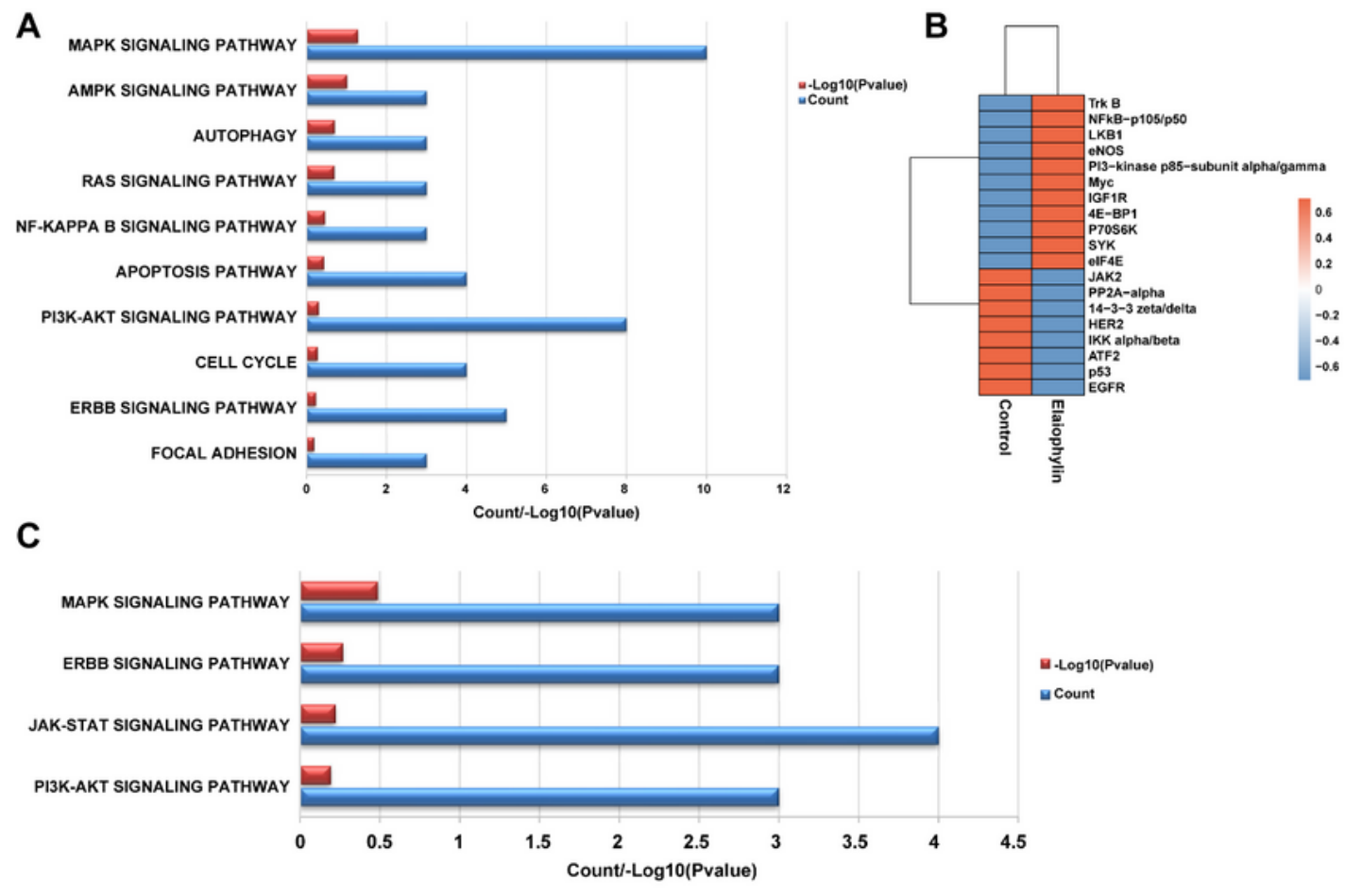

D
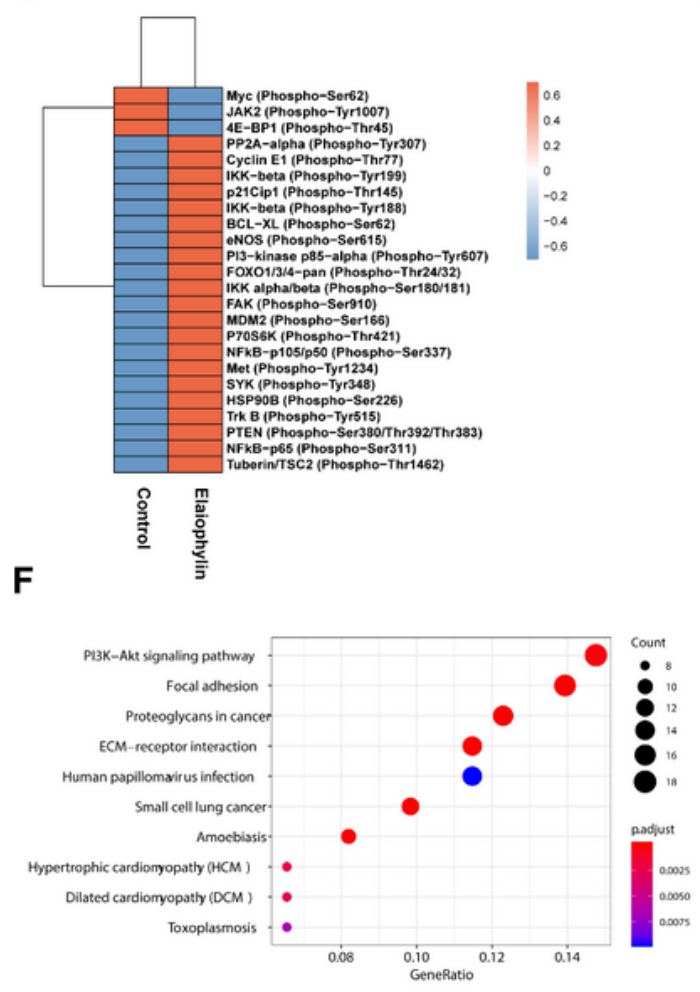

E

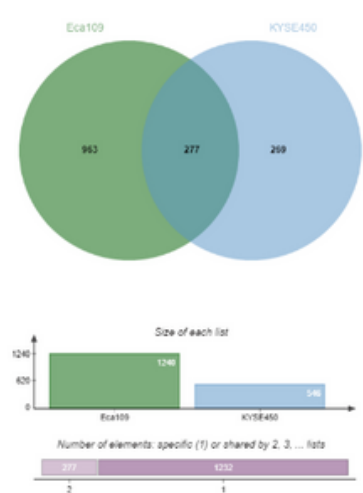

G
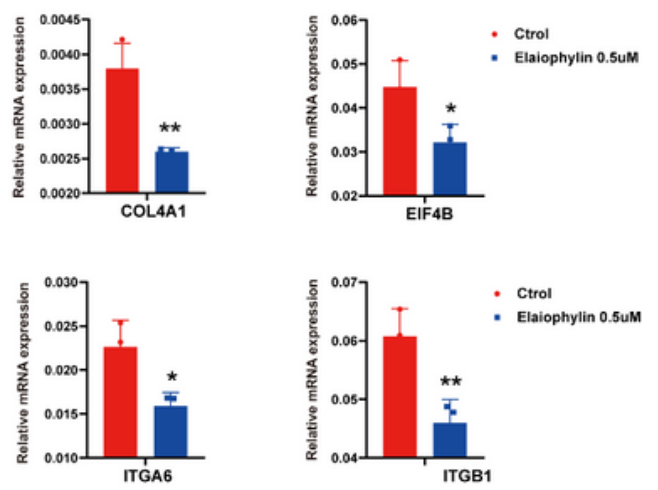

Figure 4 
Elaiophylin regulates ESCC cells through regulating proteins related to PI3K/AKT signaling pathway. (A) KEGG analysis of the downregulated proteins. (B) The differential expression proteins enrichment in the PI3K/AKT signaling pathway. (C) KEGG analysis of the downregulated phosphorylated proteins. (D) The differential phosphorylated proteins enrichment in the PI3K/AKT signaling pathway. (E) Venn diagram showed the overlaps the downregulated genes both in Eca109 and KYSE450 cells exposed to Elaiophylin. (F) KEGG analysis of the down DEGs. (G) The mRNA levels of COL4A1, EIF4B, ITGA6, ITGB1 in Eca109 was quantified by qRT-PCR normalized to Actin. The expression level levels were calculated using 2$\triangle \triangle \mathrm{CT}$ method. Error bars represent the mean $\pm S E M$. Error bars represent the mean $\pm S E M$. Statistical analysis was generated from unpaired t-test, asterisks denote statistical significance as * $\mathrm{p} \otimes 0.05,{ }^{\star *} \mathrm{p} \otimes 0.01$. 


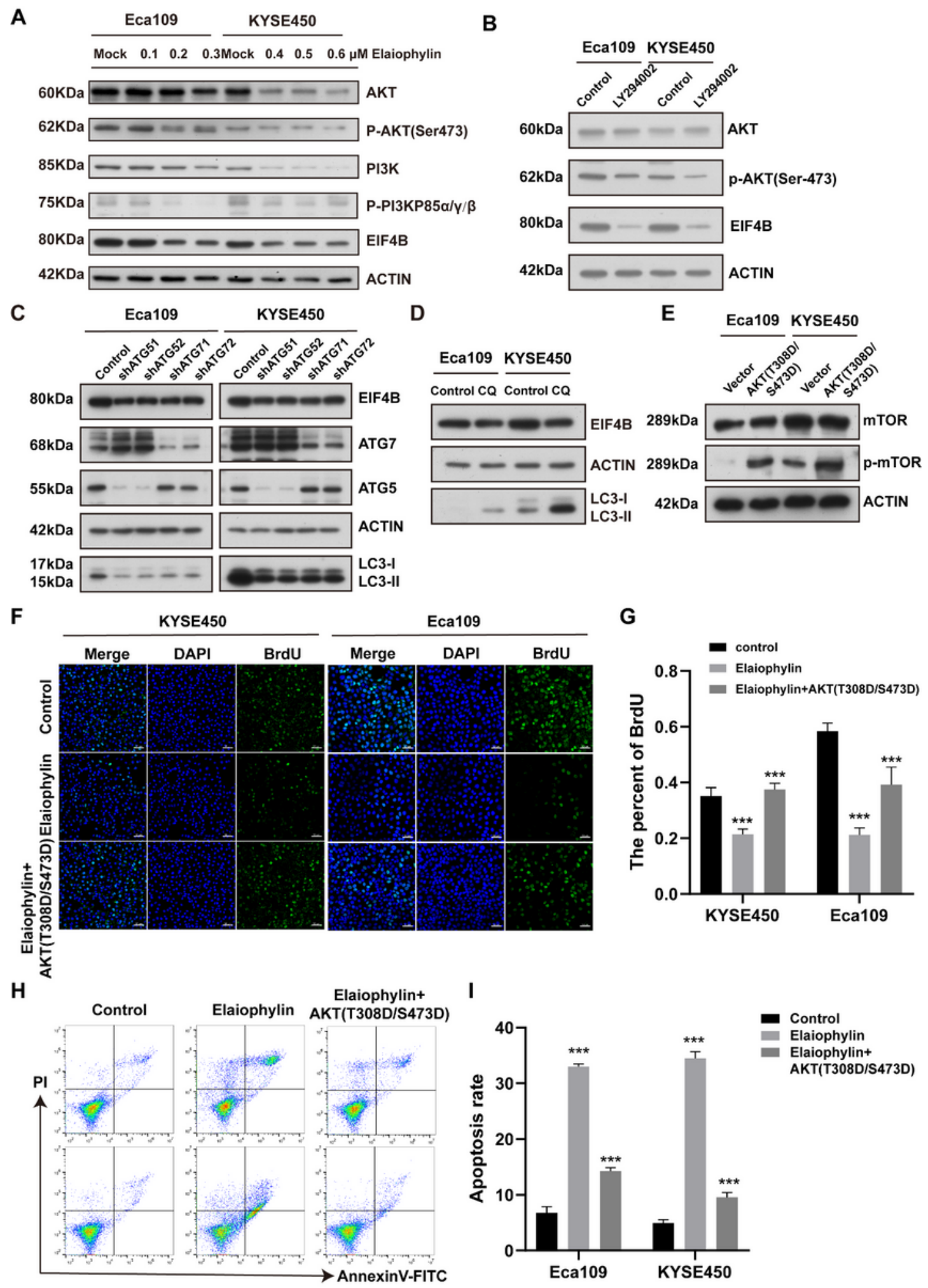

Figure 5

Elaiophylin might suppress the expression of EIF4B via PI3K/AKT signaling pathway. (A) The protein level of AKT, p-AKT, PI3K, p-PI3K, EIF4B in Eca109 and KYSE450 cells treated by different concentration of Elaiophylin were analyzed by Western blot assay. The grouping the gels cropped from different gels and same exposures. In both cell lines, the AKT, p-AKT, PI3K, p-PI3K, EIF4B protein were consistently downregulated by Elaiophylin. ACTIN was used as loading control. (B) The protein level of AKT, p-AKT, 
EIF4B was explored by western blot assay after treating with LY294002. ACTIN was used as loading control. (C) The expression of EIF4B was detected by western blot assay after shATG5 and shATG7. ACTIN was used as loading control. (D) The expression of EIF4B was detected by western blot assay after treating with CQ. ACTIN was used as loading control. (E) The expression of mTOR and p-mTOR was detected by western blot assay after transfection with AKT(T308D/S473D). ACTIN was used as loading control. (F) IHC of BrdU was used to detect the proliferation rate of Eca109 and KYSE450 cells across different groups. (G) Statistical analysis of the BrdU percent across different groups. (H) The apoptosis rate was measured by Annexin V/PI in Eca109 and KYSE450 cells. Cells with Annexin V-FITC positive and PI negative or both Annexin V-FITC and PI positive were considered apoptotic. (I) Statistical analysis of the apoptosis rate across different groups. Error bars represent the mean $\pm S E M$. Statistical analysis was

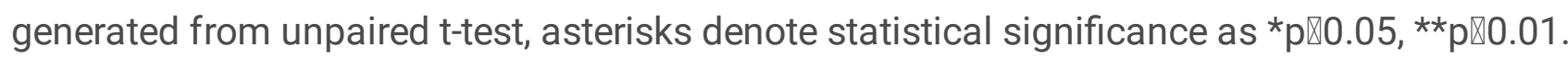


A

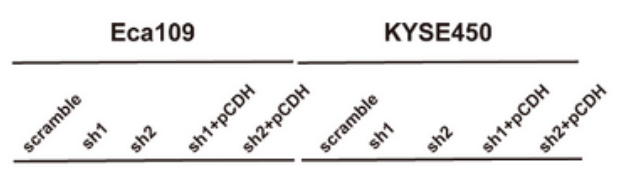

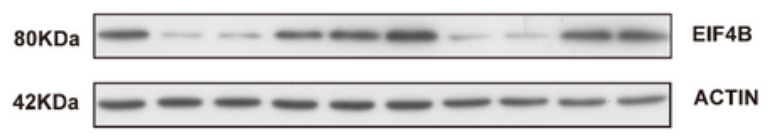

C
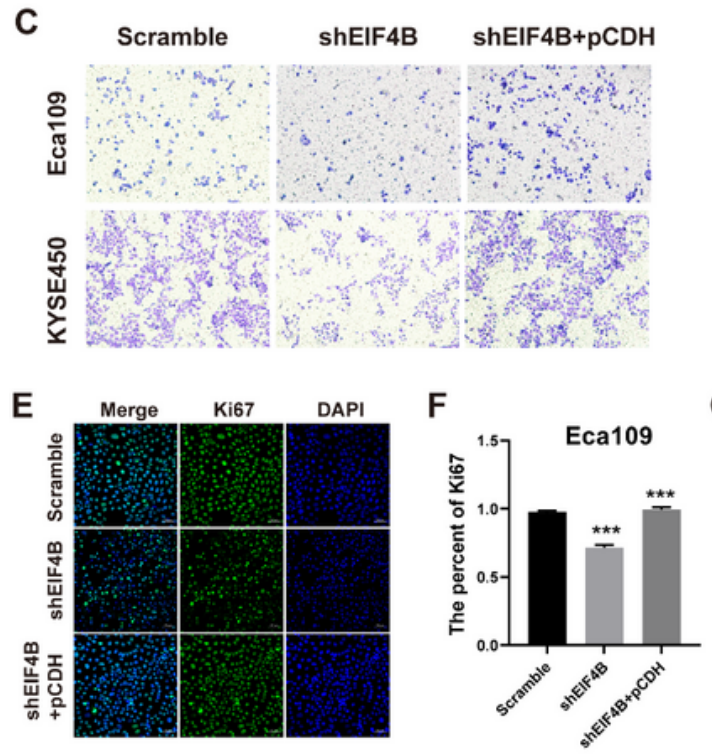

G

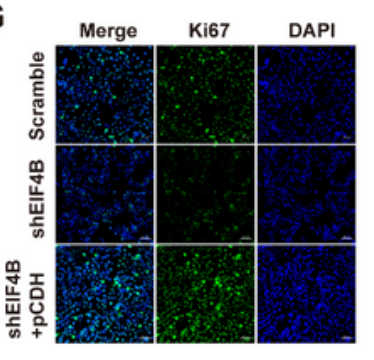

D
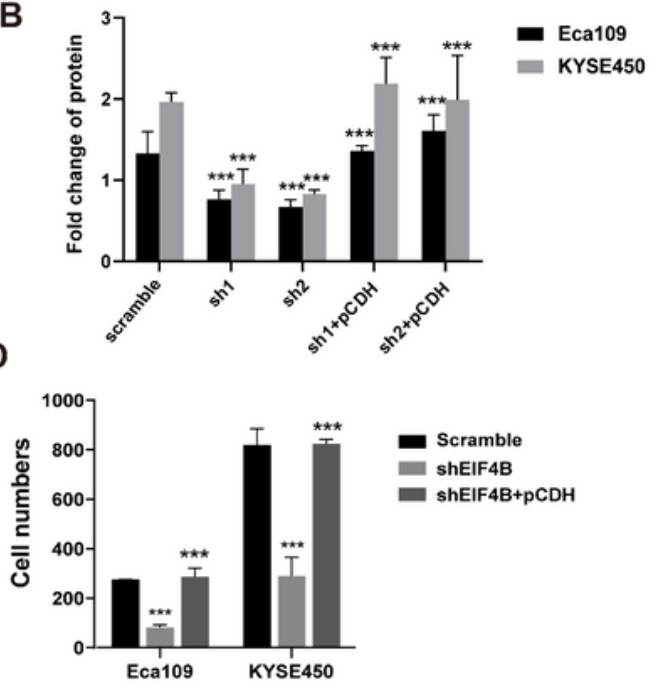

H

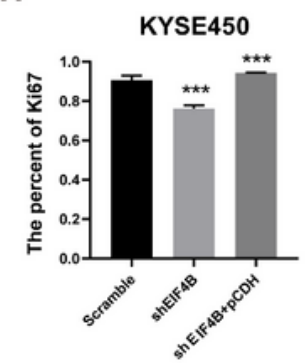

I

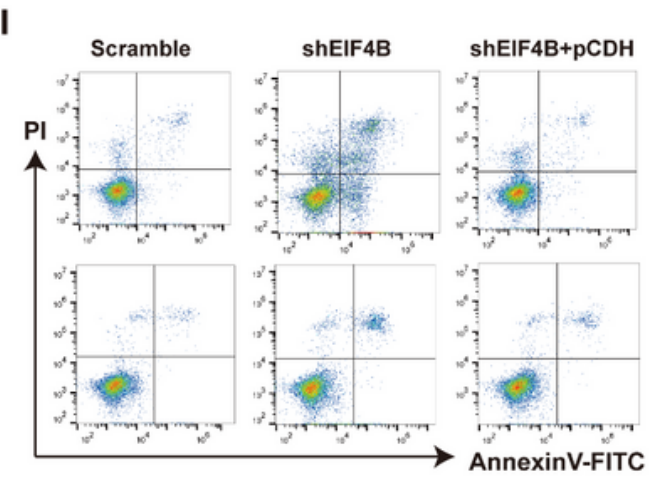

J

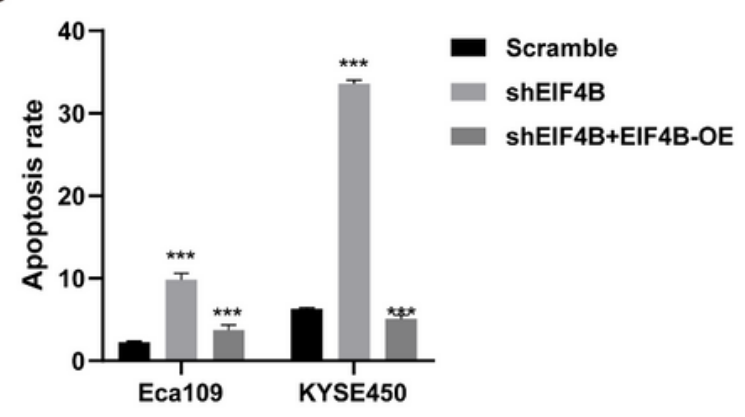

K

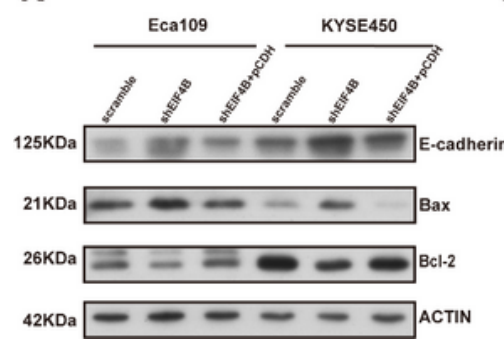

L
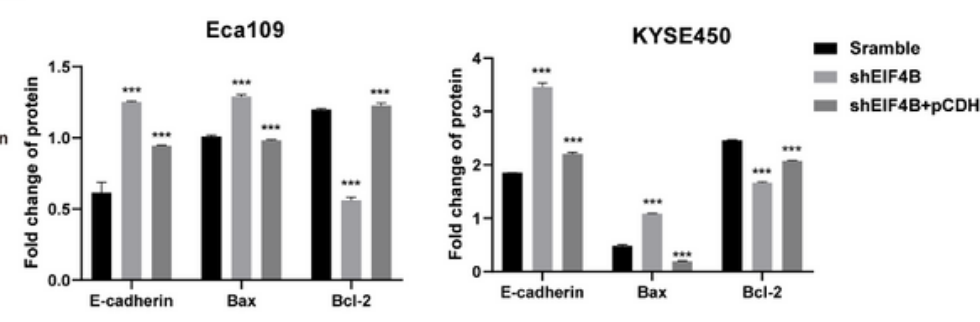

Figure 6

Knockdown of EIF4B suppresses the proliferation, migration of ESCC cells and promotes apoptosis. (A) Western blot examined the protein expression of EIF4B across different groups. (B) Statistical analysis of the protein expression of EIF4B. Error bars represent the mean \pm SEM. (C) Transwell assay measured the migration ability across different groups. (D) Statistical analysis of the cell numbers migrated to the other side of the chamber. Error bars represent the mean \pm SEM. $(E, G)$ IHC of ki67 was used to detect the 
proliferation rate of Eca109 and KYSE450 cells across different groups. $(F, H)$. Statistical analysis result of the IHC showed that the ki67 expression level was downregulated in the Elaiophylin group compared with the control, and could be reversed by EIF4B-OE. Error bars represent the mean \pm SEM. (I) The apoptosis rate was measured by Annexin V/PI in Eca109 and KYSE450 cells. Cells with Annexin V-FITC positive and PI negative or both Annexin V-FITC and PI positive were considered apoptotic. (J) Statistical analysis of the apoptosis rate across different groups. Error bars represent the mean $\pm S E M$. (K) The protein level of E-cadherin, Bax, Bcl-2 was measured in Eca109 and KYSE450 cells across different groups. The E-Cadherin, Bax, Actin was cropped from different parts of the same gel, and Bcl-2 was from different gels and from the same exposures. (L) Statistical analysis of the protein expression across different groups. Error bars represent the mean \pm SEM. Statistical analysis was generated from unpaired $t-$ test, asterisks denote statistical significance as $* \star p \otimes 0.001$. 
A
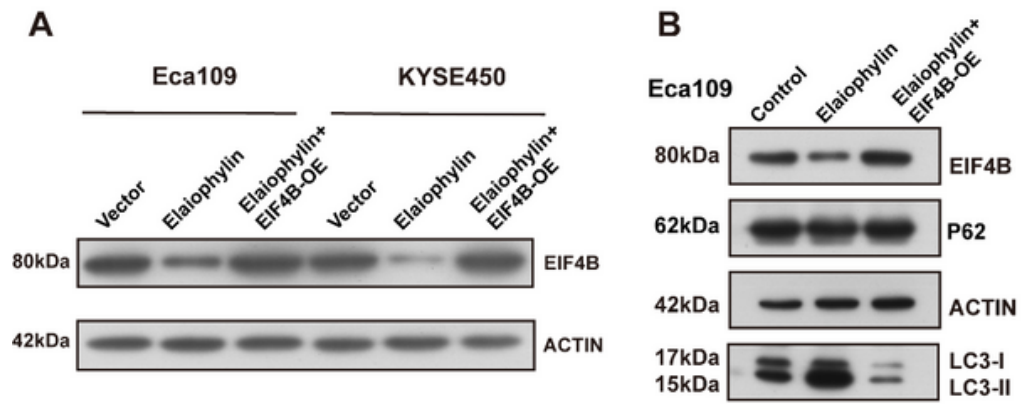

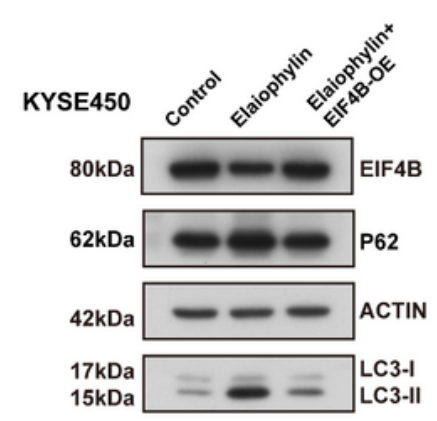

C

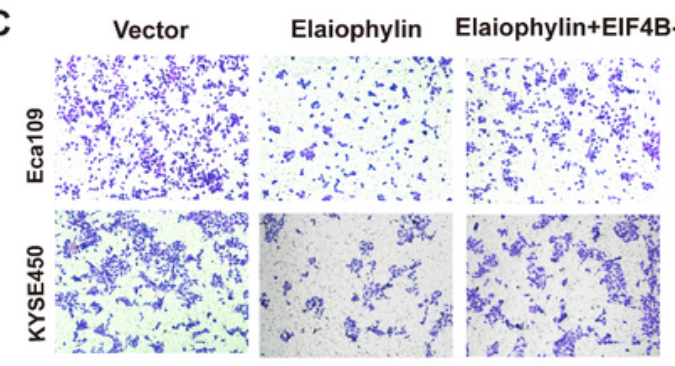

E

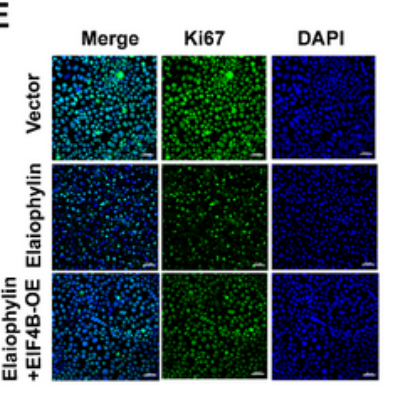

$\mathbf{F}$

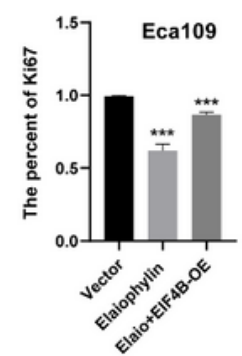

言
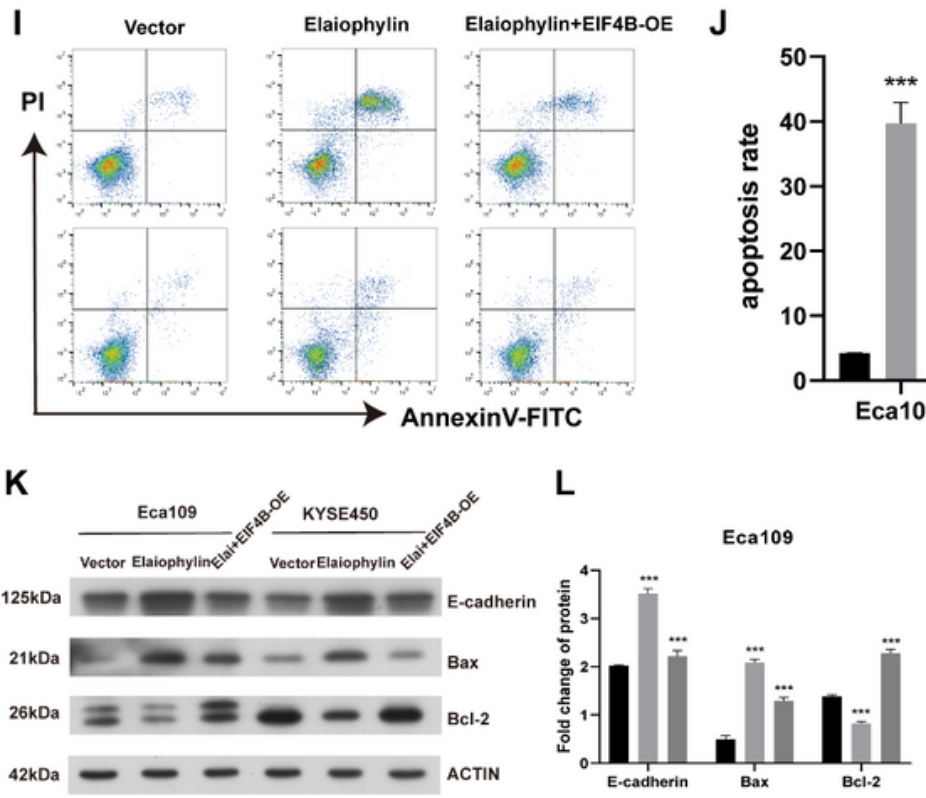

H
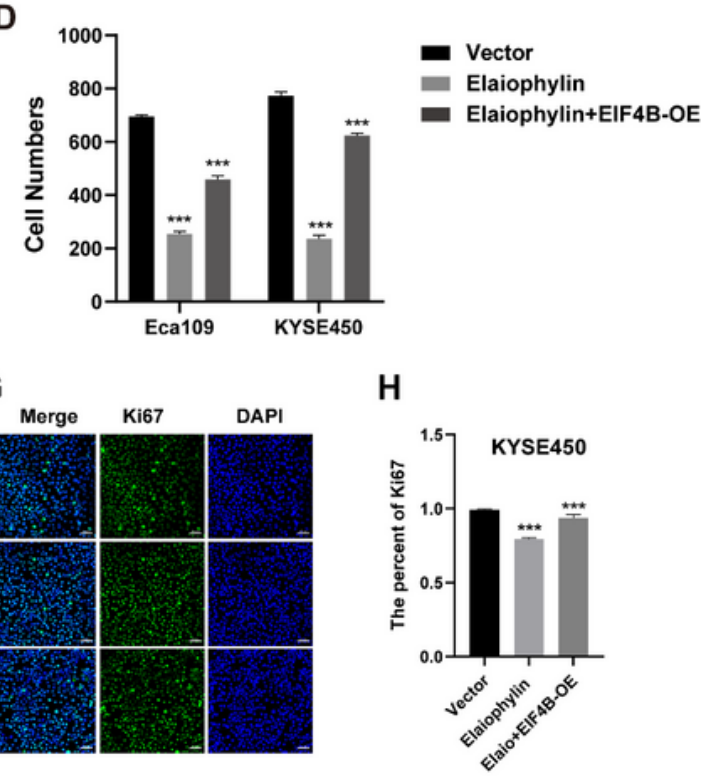

G
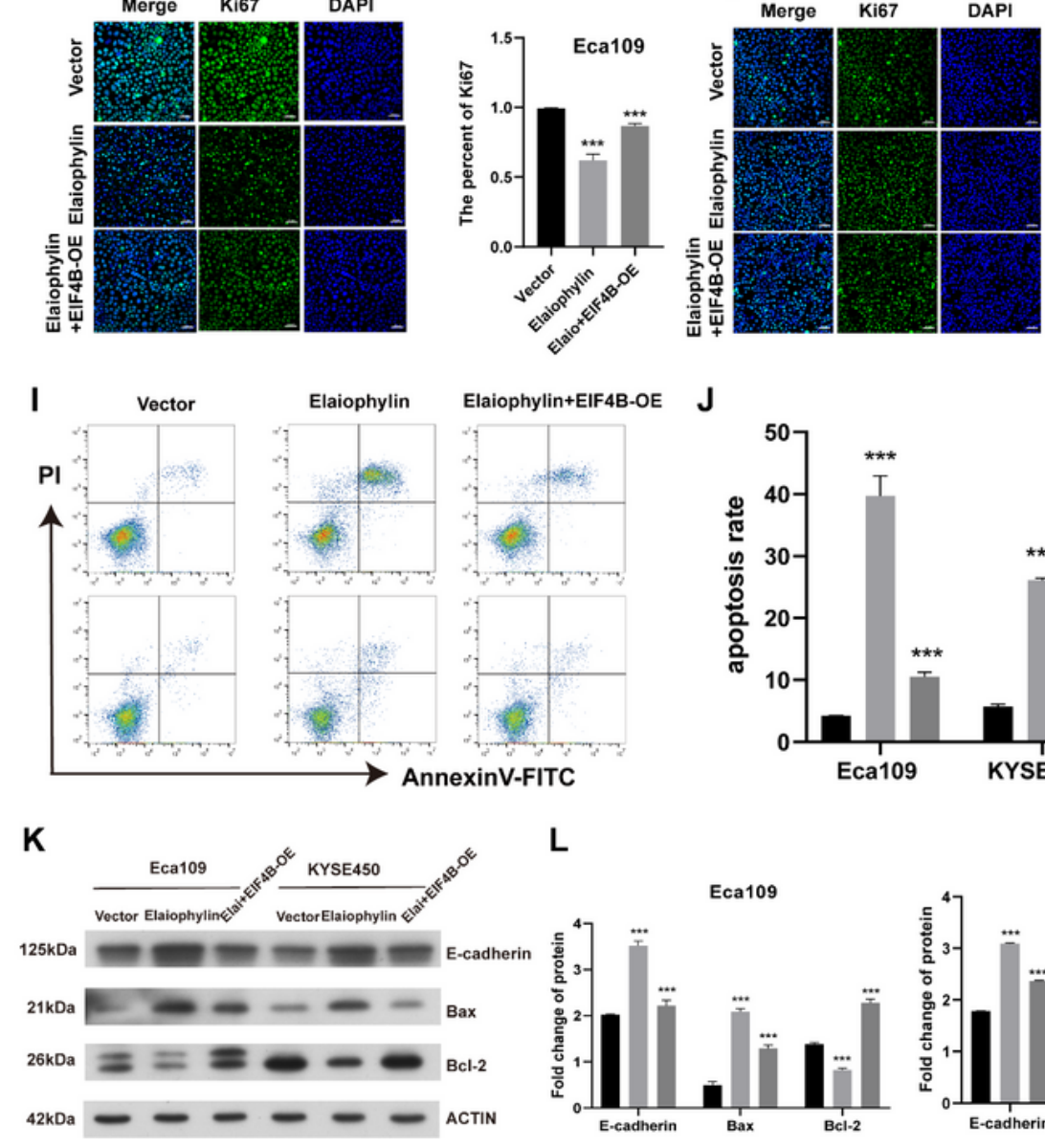

L
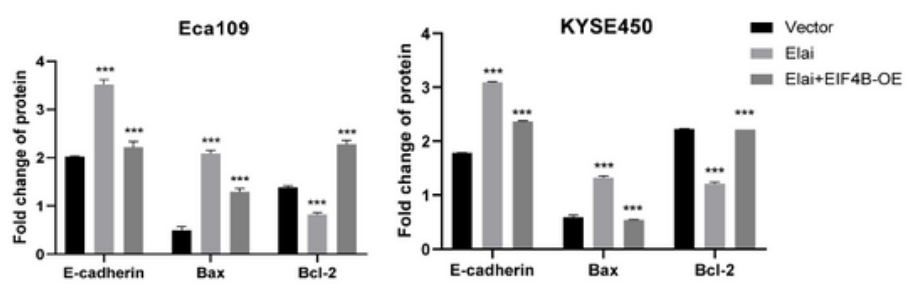

Figure 7

Upregulation of elF4B could rescued the phenotype on ESCC cells caused by Elaiophylin. (A) Western blot examined the protein expression of EIF4B among different groups. (B) Western blot assay explored the protein expression of p62 and LC3 among different groups. (C) Transwell assay measured the migration ability among different groups. (D) Statistical analysis of the cell numbers migrated to the other side of the chamber. Error bars represent the mean \pm SEM. $(E, G) I H C$ of ki67 was used to detect the proliferation 
rate of Eca109 and KYSE450 cells among different groups. $(F, H)$. Statistical analysis result of the IHC showed that the ki67 expression level was downregulated in the Elaiophylin group compared with the control, and could be reversed by EIF4B-OE. Error bars represent the mean \pm SEM. (I) The apoptosis rate was measured by Annexin V/PI in Eca109 and KYSE450 cells. Cells with Annexin V-FITC positive and PI negative or both Annexin V-FITC and PI positive were considered apoptotic. (J) Statistical analysis of the apoptosis rate among different groups. Error bars represent the mean $\pm S E M$. $(K)$ The protein level of Ecadherin, Bax, Bcl-2 was measured in Eca109 and KYSE450 cells among different groups. The E-Cadherin, Bax, Actin was cropped from different parts of the same gel, and Bcl-2 was from different gels and from the same exposures. (L) Statistical analysis of the protein expression among different groups. Error bars represent the mean $\pm S E M$. Statistical analysis was generated from unpaired t-test, asterisks denote statistical significance as **p $₫ 0.001$.

A

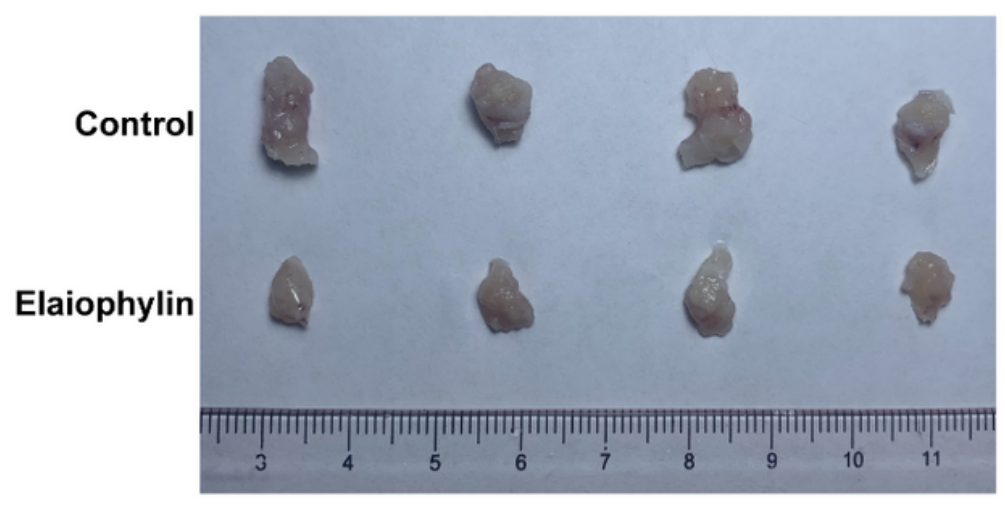

C

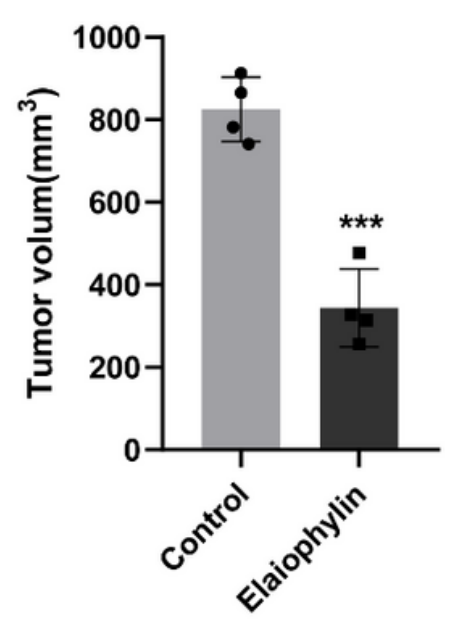

B

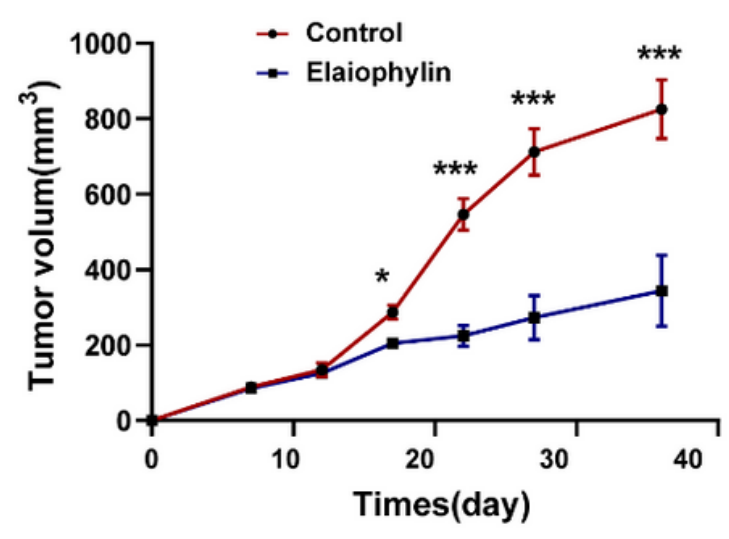

E

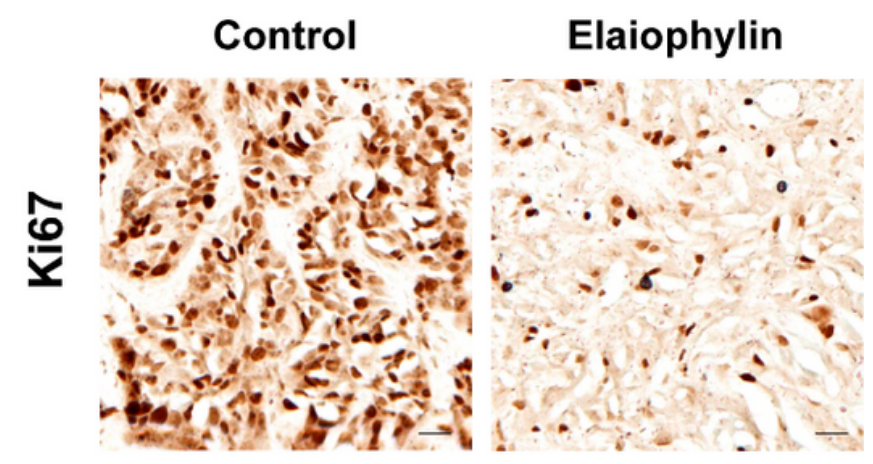

\section{Figure 8}

Elaiophylin inhibit the tumor growth of esophageal cancer in vivo. (A) The different groups of tumor were removed from the nude mice. (B) The volume of tumor of different groups in a time dependent manner. (C) The volume of tumor of different groups. (D) The weighte of tumor of different groups. (E) The IHC of 
Ki67 of different groups. the scale bar was $50 \mu \mathrm{m}$. Error bars represent the mean $\pm S E M$. Statistical analysis was generated from unpaired t-test, asterisks denote statistical significance as $\star * p \otimes 0.001$. 